This manuscript is accepted for publication in ADVANCES IN GEOPHYSICS.

The manuscript has undergone peer-review already but has no ,Peerreviewed Publication DOI' link yet. As soon as possible the link will be made available. Please feel free to contact the author; I welcome feedback. 
1 Revised submission to Advances in Geophysics

2 Rotational Ground Motion Measurements for Regional Seismic Moз ment Tensors: a Review

4 Corresponding author:

5 Donner, Stefanie

6 Address: Bundesanstalt für Geowissenschaften und Rohstoffe (BGR), GeoZentrum Han-

7 nover, Stilleweg 2, 30655 Hannover, Germany

8 E-mail: stefanie.donner@bgr.de

9 Telefon: +495116433904

10 Hannover, 8th June 2021 


\section{${ }_{11}$ Contents}

12 List of acronyms and symbols 3

\begin{tabular}{lll}
\hline 13 & Abstract & 5
\end{tabular}

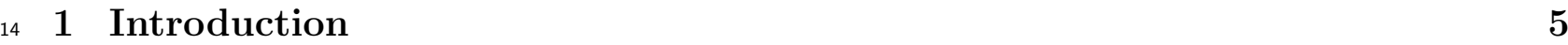

\begin{tabular}{|lr}
2 A brief mathematical overview & 8
\end{tabular}

$2.1 \quad$ Mathematical description of the point source . . . . . . . . . . . . . . . . 9

2.1 .1 Definition of the seismic moment tensor . . . . . . . . . . . . . . 9

2.1 .2 Geometry of the seismic moment tensor. . . . . . . . . . . . . . . 11

$2.1 .3 \quad$ Decomposition of the seismic moment tensor . . . . . . . . . . . . . . 14

2.2 Introduction to rotational ground motion . . . . . . . . . . . . . . 17

2.2 .1 Fundamental theory . . . . . . . . . . . . . . . 17

2.2 .2 Measuring rotational ground motions $\ldots \ldots \ldots$. . . . . . . . . 19

2.2 .3 Rotations due to a double-couple point source . . . . . . . . . . . . . 21

$2.2 .4 \quad$ Strain-rotation coupling and local side effects . . . . . . . . . . . 21

$3 \quad$ Benefits of rotational ground motions for regional seismic moment tensors 22

$3.1 \quad$ General benefits for the resolution of the moment tensor components . . . . 23

3.2 Influence of the receiver distribution . . . . . . . . . . . . . . . . . . . 26

$3.3 \quad$ Influence of frequency range and structural model . . . . . . . . . . . . . . . 30

3.4 Resolution of the centroid depth . . . . . . . . . . . . . . . . . . . . . . 33

3.5 Resolvability of the tectonic mechanism and DC/non-DC parts . . . . . . . . 34

3.6 Influence of noise . . . . . . . . . . . . . . . . . . . . . . . . . . 35

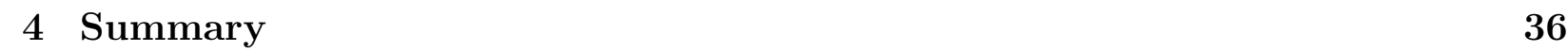

\begin{tabular}{lll}
\hline 5 & Outlook & 37
\end{tabular}

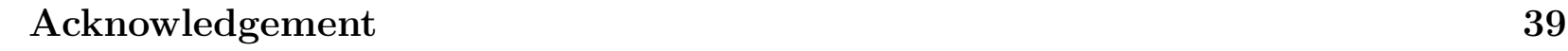

35 References $\quad 39$ 


\title{
List of acronyms and symbols
}

\author{
Acronyms \\ $1 \mathrm{D} / 2 \mathrm{D} / 3 \mathrm{D} \quad 1-/ 2-/ 3$-dimensional \\ $3 \mathrm{C} / 6 \mathrm{C} \quad 3 / 6$ components of waveform data: translation vs. translation+rotation \\ CLVD compensated linear vector dipole part of source mechanism \\ DC double-couple (tectonic) part of source mechanism \\ DEV deviatoric part of source mechanism \\ GF \\ Green's function \\ IG \\ Shannon's criterion of information gain \\ INSN \\ Iranian National Seismic Network \\ ISO \\ isotropic (volumetric) part of source mechanism \\ kde \\ (Gaussian) kernel density estimation \\ pdf \\ (prior/posterior) probability density function
}

42

45

\section{Symbols}

$\hat{\mathbf{r}}, \hat{\boldsymbol{\theta}}, \hat{\boldsymbol{\phi}} \quad$ unit vectors of spherical polar coordinate system

51

$\mathbf{A}^{P}, \mathbf{A}^{S}, \mathbf{A}^{R}$ radiation pattern of translational P- and S-wave, and rotational S-wave

52

$\mathbf{a}_{i}, e_{i}$

eigenvectors and -values of the moment tensor

53

d

displacement (or slip) vector on the rupture plane

54

$\mathrm{G}, G_{i j}, G_{i j, k}$

tensor of Green's functions and its spatial derivatives

55

$\mathbf{J}$

strain-rotation coupling vector

56

$\mathbf{M}, M_{j k}$

seismic moment tensor

$57 \quad \mathbf{S}_{k}$

slowness vector

58

$\mathbf{t}, \mathbf{b}, \mathbf{p}$

tension/null/pressure axis of moment tensor

59

$\mathbf{u}, u_{i}$

translational displacement field, field component

60

$\mathbf{x}, t$

location and time of observation

${ }_{61} \boldsymbol{\omega}, \omega_{i}$

rotational ground motion, field components

62

$\boldsymbol{\omega}^{F S}$

rotational ground motion at the Earth surface 


$\begin{array}{lll}{ }_{63} & \boldsymbol{\xi}, \tau & \text { location and time of source origin } \\ { }_{64} & \phi, \delta, \lambda & \text { strike, dip, and rake angles of DC part of the moment tensor; angles of } \\ { }_{65} & & \text { rupture plane and slip vector orientation } \\ { }_{66} & F_{j} & \text { total force component exerted by the source } \\ { }_{67} & f_{j} & \text { body force density component within source region, 'stress glut' } \\ { }_{68} & M_{0} & \text { seismic moment } \\ & & \text { Source time function }\end{array}$




\begin{abstract}
Seismic moment tensors are an important tool in geosciences on all spatial scales and for a broad range of applications. The basic underlying theory is established since decades. However, various factors influence the reliability of the inversion result, several of them are mutually dependent. Hence, a reliable retrieval of seismic moment tensors is still hampered in many cases, especially at regional event-receiver distances.

To sample the entire wavefield due to a seismic source we need six components: three translational and three rotational ones. Up to now, only translational ground motion recordings were used for moment tensor retrieval, missing out valuable information.

Using rotational in addition to the classical translational ground motions during waveform inversion for moment tensors mainly adds information on the vertical displacement gradient to the inversion problem. Furthermore, having available six instead of only three components per receiver location provides additional constraints on the sampling of the radiation pattern.

As a result, the moment tensor components are resolved with higher precision and accuracy, even when the number of recording receivers is considerably reduced. Especially, components with a dependence to depth as well as the centroid depth can benefit significantly from additional rotational ground motion.

Up to the time of writing this review only a few studies are published on the topic. Here, I summarise their findings and provide an overview over the possible capabilities of including rotational ground motion measurements to waveform inversion for seismic moment tensor retrieval.
\end{abstract}

\title{
1 Introduction
}

In an effort to mathematically describe the source processes during an earthquake, mainly three models have emerged over time: seismic moment tensors, kinematic, and dynamic source descriptions (Aki and Richards, 2002). They differ in their underlying assumptions and applied simplifications as well as in their information on the source processes. Seismic moment tensors assume all energy release in one point in space described by a system of body forces acting at that point. We can derive information on source geometry, strength and composition. The kinematic source model assumes a time-dependent displacement field but without considering the stresses and forces responsible for it. We get information on the rupture dimension and propagation as well as slip direction and slip rate function in addition to geometry and strength. The most complex model is the dynamic source model which aims at a complete physical understanding of the rupture physics including initial and boundary conditions for the stresses and forces involved as well as material properties. It is based on solving the elastodynamic equation of motion and, thus, is quite expensive in 
storage and computational resources.

Seismic moment tensors are an integral and essential part of geosciences (D'Amico, 2018). They help us to understand the mechanics of single earthquakes or earthquake sequences (e. g. Kanamori and Given, 1982; Dreger and Helmberger, 1991; Braunmiller et al., 2002, Krüger and Scherbaum, 2014). Using a comprehensive catalogue of moment tensor solutions, they provide useful insight into the seismotectonics of a region (e.g. Herrmann et al., 2011; Kinscher et al., 2013; Dahm et al., 2018; Schippkus et al., 2020). Also, such a catalogue of moment tensors can be inverted again to obtain information on the stress condition of the upper crust (e.g. Gephart and Forsyth, 1984; Heidbach et al., 2018). Lessons learned from seismotectonic and crustal stress studies in turn are a fundamental base for seismic hazard studies. In Tsunami warning systems seismic moment tensors are a crucial input to estimate the likeliness of such a threat within a few minutes after a large earthquake (e.g. Kanamori and Rivera, 2008; Inazu et al, 2016).

While we need some prior knowledge about Earth structure to determine moment tensors (see Sec. 2.1.1) they also help us to gain an improved estimate on underground structures. In tomographic studies, moment tensors are used to simulate seismic waveforms and study their distortions while travelling through the underground. These distortions provide direct evidence for structural details (e.g. Toyokuni and Takenaka, 2006; Nissen-Meyer et al. 2014; Shi et al. 2018).

In contrast to focal solutions from first motion body wave polarities, seismic moment tensors contain information beyond the pure shear motion. Hence, they are a useful tool for source discrimination. Possible applications are the identification of an explosion, for example in the frame of the Comprehensive Nuclear Test-Ban Treaty (e.g. Gaebler et al., 2019), the interpretation of volcanic signals (e. g. Nettles and Ekström, 1998, Legrand et al., 2000), and the monitoring of induced seismicity for example at geothermal facilities, mining areas or within oil/gas production fields (e.g. Dahm et al., 2007; Cesca et al., 2013; Sen et al., 2013; Kühn et al., 2020).

Almost 60 years ago, Haskell (1964) presented equations for the radiation pattern of surface waves from double-couple (DC, see section 2.1.3 point sources in a layered medium. In 1970 Gilbert provided 'a very compact and simple representation for the transient response of the Earth to earthquakes', the first definition of the seismic moment tensor. He used it to calculate the static displacement field caused by Earth' normal mode eigenfunctions. Three years later, he extended his theory to the formulation of a linear inverse problem with six independent elements for the moment tensor (Gilbert, 1973). Dziewonski and Gilbert (1974) and Gilbert and Dziewonski (1975) further extended the theory and applied it to determine the source mechanisms of earthquakes. The seismic moment tensor is related to the inelastic processes inside the focal region only and is zero outside of it (Backus and Mulcahy, 1976 ; 
Backus, 1977a). Therefore, it is a perfect general concept to describe a variety of seismic sources.

The seismic point-source moment tensor is a low-frequency assumption, valid only below the corner frequency of the studied event (see section 2.1.1). In addition, several authors investigated early the relation between moment tensors of higher degree to seismic sources with finite extend (kinematic sources). Here, Backus (1977a), Backus (1977b), Stump and Johnson (1982), Doornbos (1982), and Silver (1983) are to be mentioned for example. However, in this study, I focus on the point-source moment tensor.

Traditionally, the waveform inversion for seismic moment tensors is further classified in global, regional, and sometimes also local application. Yet, this classification is just a proxy for different length scales and the amount of heterogeneities involved. Although, there is no strict definition, we can say that 'global' starts beyond event-receiver distances of about 1000-3000 km for moment tensor applications. The larger the event-receiver distance, a larger fraction of the wavefield passes through deeper parts of the Earth instead of the crust. The velocity structure of the Earth interior is much more homogeneous than than the crustal structure. Therefore, teleseismic recordings are easier to model, a processing step necessary in waveform inversion for moment tensors. As soon as distances become close enough that the wavefield fraction passing the highly complex structures of the crust is longer than the wavefield fraction passing deeper Earth, waveform inversion becomes more complicated. This is mainly due to the more complex waveforms compared to the teleseismic distance range. Several practical consequences for the waveform inversion can be derived from this general difference. They are explained in more detail in section 3.3 . Keeping this generalisation in mind the classification is rather twofold between 'global' and 'regional', with 'local' being a subclass of regional.

On the global scale the implementation of waveform inversion schemes are well established. Since 1982 the Global CMT Project (formerly called the Harvard CMT Project) provides a global catalogue of moment tensor solutions, comprehensive for earthquakes with magnitudes M > 5 (Dziewonski et al., 1981; Ekström et al., 2012). The determination on a regional (or even local) scale is more complicated. The stability of the waveform inversion result is affected by less well-known structural models (e.g. Šilený, 2004; Vasyura-Bathke et al., 2021), influence of theoretical and measurement noise (e. g. Sipkin, 1986; Duputel et al., 2012, Mustać et al., 2020), unfavourable event-receiver geometries (e.g. Dreger and Helmberger, 1993; Delouis and Legrand, 1999), ignored source complexities (e.g. Adamova and Šilený, 2010), and limitations of methodological approaches (e.g. Fan and Wallace, 1991; Frohlich, 1994; Julian et al., 1998; Cesca and Heimann, 2018).

The literature about the topic is so numerous that the citations given in here can cover only a small fraction of it. I hope that it provides an overview broad enough to serve as a good 
starting point for own research. Providing a fully comprehensive review on all aspects of seismic moment tensors would include the complete theory, an overview on methodological approaches for inversion as well as codes and algorithms, discussing the limits and uncertainty estimation, backlashes, etc. That is a book-filling task and beyond the scope of this paper. Therefore, this review concentrates on some practical aspects during waveform inversion for seismic moment tensors in the regional distance range. These are probably also applicable to the local distance range because of similar reasons for complexities. The main focus is the integration of rotational ground motion recordings into the waveform inversion and how they can improve the reliability of the resulting seismic moment tensor.

A short comment on the wording: very often I read about 'moment tensor inversion'. This is logically not entirely correct. Moment tensors are the output of the inversion. The inputs are waveforms, i. e. recordings of the wavefield or seismograms. Therefore, the logically correct phrasing should be 'waveform inversion for seismic moment tensors' or 'moment tensor retrieval or determination'.

The paper is structured in two main parts. After briefly introducing the theory of the seismic moment tensor and rotational ground motions in Section 2, Section 3 provides a review on the results of waveform inversion including rotational ground motions for seismic moment tensors. It is followed by a summary in Section 4 and an Outlook in Section 5.

\section{A brief mathematical overview}

In the following, I provide a brief overview of the theoretical background of the seismic moment tensor as well as rotational ground motions. The information given are restricted to what is necessary to understand this review article. For more in-depth theory about the seismic moment tensor, I suggest for beginners Jost and Herrmann (1989), Krüger and Bock (2009), Cesca and Heimann (2013), and Dahm and Krüger (2014). More advanced readers may prefer Udías, Madariaga, and Buforn (2014) and Aki and Richards (2002). An introduction into rotational ground motions for beginners can be found in Cochard et al. (2006), Li and van der Baan (2017), and Igel et al. (2015). For more advanced readers I suggest Aki and Richards (2002), Schmelzbach et al. (2018) and Sollberger et al. (2020).

In the following, tensors appear in bold face (for example $\mathbf{u}$ for vectors and $\mathbf{M}$ for matrices). Subscripts (for example $u_{i}$ ) indicate components of tensors with $i=x, y, z$ being the three space axes. Einstein's summation convention applies on repeated indices. 


\subsection{Mathematical description of the point source}

\subsubsection{Definition of the seismic moment tensor}

For a general elastodynamic source, the linear relation between the observed displacement field component $u_{i}$ at observation point $\mathbf{x}$ and time $t$ and the distribution of equivalent body force densities $f_{j}$ within the source region $\boldsymbol{\xi}$ at origin time $\tau$ is given by (Gilbert, 1970; Aki and Richards, 2002):

$$
u_{i}(\mathbf{x}, t)=\iiint_{V} G_{i j}(\mathbf{x}, t-\tau ; \boldsymbol{\xi}, 0) * f_{j}(\boldsymbol{\xi}, t) d^{3} \xi
$$

Displacement and traction are omitted. The * marks a temporal convolution. $G_{i j}$ are the Green's functions (GF) defined as the response of the medium to a unidirectional unit $\delta$ Dirac impulse in space and time. They provide the $i$-th component of displacement at place $\mathbf{x}$ and time $t$ due to a force in direction $j$ at place $\boldsymbol{\xi}$ and time $\tau$. Because the boundary conditions at $t<\tau$ are time-independent, the GFs depend only on the difference between origin and observation time $t-\tau$ but not on the origin time $\tau$ itself.

The spatial integral in Eq. 1 can be simplified by Taylor series expansion around the source $\boldsymbol{\xi}_{0}$ as reference point. This reference point is defined as the centroid location of the source, the point of highest energy release. Usually, it does not coincide with the hypocenter, the point where the rupture starts. Applying the point source assumption, we assume that the GF do not change (much) within the source volume. That is, the considered wavelength must be larger than the source dimension. Then, we only need to consider the first two terms of the Taylor series and the equation modifies to:

$$
u_{i}(\mathbf{x}, t)=G_{i j}\left(\mathbf{x}, t-\tau ; \boldsymbol{\xi}_{0}, 0\right) * F_{j}(t)+G_{i j, k}\left(\mathbf{x}, t-\tau ; \boldsymbol{\xi}_{0}, 0\right) * M_{j k}(t)
$$

$F_{j}(t)$ is the total force exerted by the source. It is defined as the volume integral of the bodyforce density $f_{j}(\boldsymbol{\xi}, t)$ arising from the difference between the model stress and the actual physical stress when describing the physical, non-linear source processes by an equivalent force system (Julian et al., 1998). Backus and Mulcahy (1976) called this body-force density the 'stress glut'. Omitting effects of gravitation and mass advection, the stress glut vanishes outside the source medium and, hence, the total force $F_{j}(t)$ is zero. It remains:

$$
u_{i}(\mathbf{x}, t)=M_{j k}(t) * G_{i j, k}\left(\mathbf{x}, t-\tau ; \boldsymbol{\xi}_{0}, 0\right)
$$

This is the basic equation for the waveform inversion for seismic moment tensors. The (linearised) forward problem is given by $\mathbf{u}=\mathbf{G m}$. It can be further simplified when we formally rewrite the tensor of GFs to $G_{i j}=G_{i j}(\mathbf{x}(\xi), t(\xi))$. Then, the spatial derivative in 
Eq. 3 turns to:

$$
\begin{aligned}
G_{i j, k} & =\frac{\delta}{\delta \xi_{k}} G_{i j} \\
& =\frac{\delta G_{i j}}{\delta x_{n}} \frac{\delta x_{n}}{\delta \xi_{k}}+\frac{\delta G_{i j}}{\delta t} \frac{\delta t}{\delta \xi_{k}}
\end{aligned}
$$

The first term of the right hand side is the near-field term and can be neglected at larger distances. The second term is the far-field. The second factor of the second term is the slowness vector $s_{k}=\frac{\delta t}{\delta \xi_{k}}$. Omitting near-field effects, equation 3 turns to:

$$
\begin{aligned}
u_{i}(\mathbf{x}, t) & =M_{j k}(t) * \dot{G}_{i j}(\mathbf{x}, t) s_{k} \\
& =\dot{M}_{j k}(t) * G_{i j}(\mathbf{x}, t) s_{k}
\end{aligned}
$$

with the dot representing temporal derivative which can be interchanged between the GFs and the moment tensor.

We assume that all components of the moment tensor $\mathrm{M}_{j k}(t)$ have the same time dependence which corresponds to the source time function $S(t)$. With the point source assumption, the source time function is modelled as a Dirac $\delta$-pulse and, thus, becomes unity in the far-field. This approach is valid as long as the real source duration is shorter than the shortest periods (that is highest frequencies) considered during inversion. With another Taylor expansion of the GFs around the centroid time $\tau_{0}$ and again neglecting terms of higher order we obtain (Dahm, 1993; Dahm and Krüger, 2014):

$$
\begin{aligned}
u_{i}(\mathbf{x}, t) & =M_{j k} \cdot\left[\dot{S} * G_{i j}(\mathbf{x}, t) s_{k}\right] \\
& =M_{j k} \cdot G_{i j, k}(\mathbf{x}, t)
\end{aligned}
$$

The second factor in this equation is a rephrase of the spatial derivatives of the far-field GFs with respect to the centroid.

From the seismic moment tensor, we not only obtain information about the physical source processes (see section 2.1.3 but also on the strength of the source. The seismic moment is defined directly on the moment tensor components Silver and Jordan (1982):

$$
M_{0}=\sqrt{\frac{\sum M_{k j}^{2}}{2}}
$$

It has the unit of energy $(\mathrm{Nm})$ and we can derive the moment magnitude from it. In addition, 
the waveform inversion for the seismic moment tensor provides us with an estimate for the centroid depth. The pre-calculated GFs needed for the inversion are based on a specific depth. In practice, the waveform inversion is repeated with GFs for different depths and the inversion with the best residual is assumed to be in the correct depth.

\subsubsection{Geometry of the seismic moment tensor}

The seismic moment tensor $\mathrm{M}_{i j}$ is the most general description of equivalent body forces for a seismic point source. It is a tensor of second order with nine components (Fig. 1). Each represents the strength (moment) of an elementary force couple.
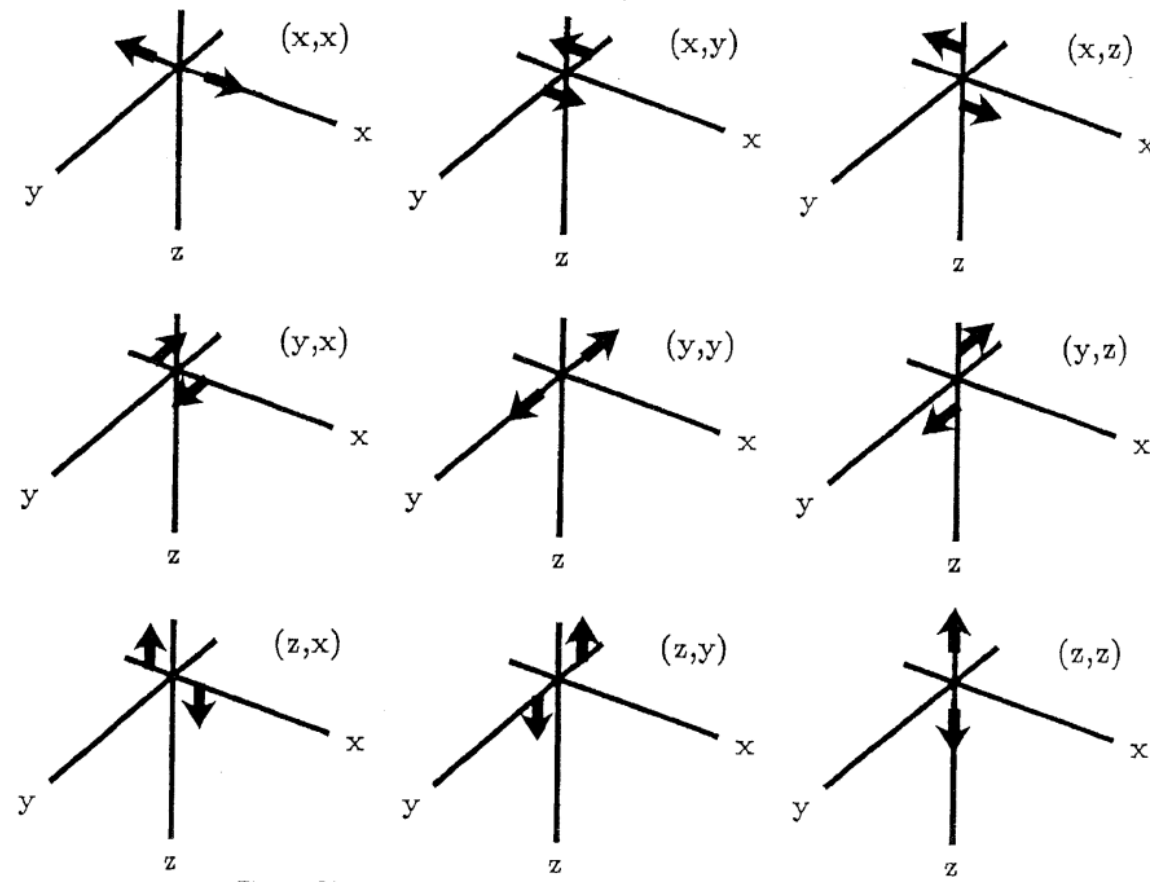

Figure 1: The nine components of the seismic moment tensor representing generalised force couples (from Jost and Herrmann, 1989).

The diagonal elements represent linear dipoles causing no torque. The off-diagonal elements correspond to force couples ('double-couples'). They represent shear dislocation with three different orientations. They can be connected to the displacement (or slip) vector $\mathbf{d}$ on the rupture plane and its normal vector $\mathbf{n}$ :

$$
M_{j k}=\mu A\left(d_{k} n_{j}+d_{j} n_{k}\right)
$$

with $\mu$ and $A$ being the shear modulus of the source area and the area of the rupture plane, respectively (Fig. 2). Because switching $\mathbf{d}$ and $\mathbf{n}$ has no consequences on the resulting tensor element, it follows that the moment tensor is symmetric and, therefore, also the off-diagonal 
elements exert no torque. As a consequence, only six of the nine moment tensor components are independent which can be inverted for from wavefield recordings.

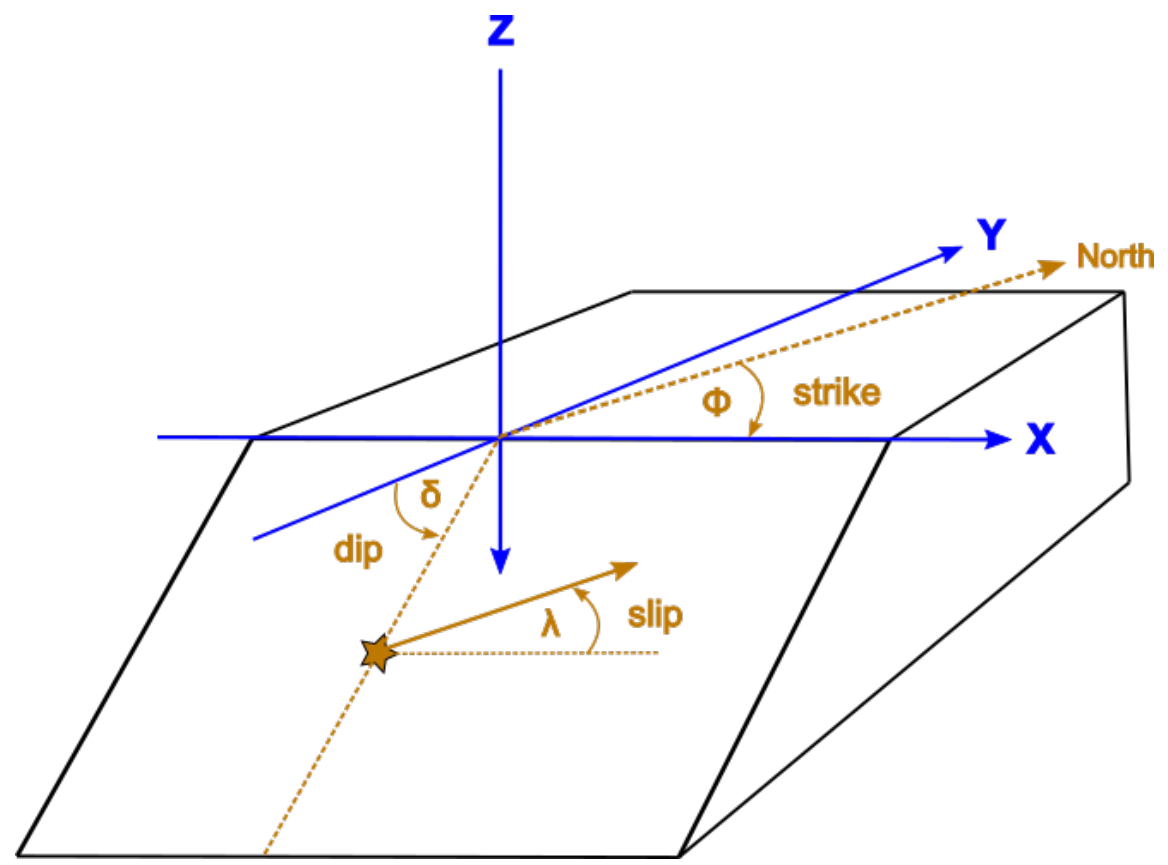

Figure 2: Block model of a shear rupture plane showing the three angles strike, dip, and slip or rake defining the orientation of the rupture plane and of the displacement (or slip) vector. These angles are related to the seismic moment tensor via Eq. 11. The star on the rupture plane marks the centroid location.

It also follows that the displacement and normal vector can not be separated from each other. Therefore, the source solution contains two nodal planes and it needs further information to distinguish the fault from the auxiliary plane. For example, these information be aftershock distribution, known fault orientation, or borehole breakouts.

In an infinite, homogeneous and isotropic medium we can describe the ground motion due to a point shear dislocation (DC) with an analytical solution. Aki and Richards (2002) have given the expressions for the vertical, radial and transversal components of the displacement $\boldsymbol{u}(\mathbf{x}, t)$ in spherical polar coordinates centered on the source (Fig. 3). These expressions include terms for the energy radiation pattern for the P- and S-wavefield in the near, intermediate, and far-field. For the far-field these radiation pattern terms are:

$$
\begin{aligned}
\mathbf{A}^{P} & =\sin 2 \theta \cos \phi \hat{\mathbf{r}} \\
\mathbf{A}^{S} & =\cos 2 \theta \cos \phi \hat{\boldsymbol{\theta}}-\cos \theta \sin \phi \hat{\boldsymbol{\phi}}
\end{aligned}
$$

The vectors $\hat{\mathbf{r}}, \hat{\boldsymbol{\Theta}}$, and $\hat{\boldsymbol{\Phi}}$ are the unit vectors of the spherical polar coordinate system in 


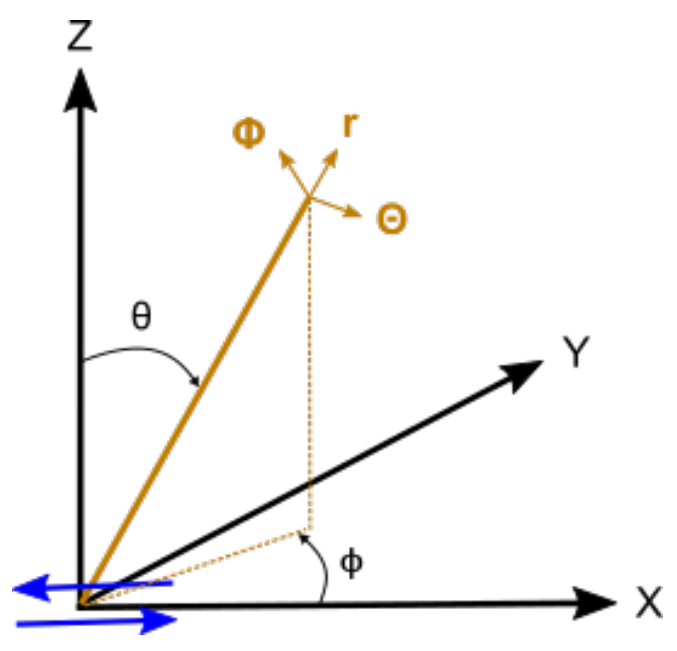

Figure 3: Cartesian (black) and spherical polar (orange) coordinates to describe doublecouple displacement components. Blue indicates a point shear dislocation in the $\mathrm{x}-\mathrm{y}$ plane related to Eqs. 9 and 19 for radiation patterns of translation and rotation.

the vertical, radial, and transverse directions, respectively (see Fig. 3). Mathematically, the moment tensor can be rotated in any coordinate system. Thus, we always find a system where the tensor comprise only three orthogonal linear force dipoles. Then, the eigenvectors of the moment tensor correspond to the principal axes which can be defined via the displacement and normal vector (Jost and Herrmann, 1989):

$$
\begin{array}{rr}
\mathbf{t}=\frac{1}{\sqrt{2}}(\mathbf{n}+\mathbf{d}) & \text { tension axis } \\
\mathbf{b}=\mathbf{n} \times \mathbf{d} & \text { null axis } \\
\mathbf{p}=\frac{1}{\sqrt{2}}(\mathbf{n}-\mathbf{d}) & \text { pressure axis }
\end{array}
$$

Using these equations, we can derive the connection between the moment tensor components $\mathrm{M}_{j k}$ and the geological faulting geometry, meaning the three angles strike $\phi$, dip $\delta$, and rake 
(or slip) $\lambda$ of a shear (DC) mechanism (Fig. 2, Jost and Herrmann, 1989):

$$
\begin{aligned}
& M_{x x}=-M_{0}\left(\sin \delta \cos \lambda \sin 2 \phi+\sin 2 \delta \sin \lambda \sin ^{2} \phi\right) \\
& M_{y y}=M_{0}\left(\sin \delta \cos \lambda \sin 2 \phi-\sin 2 \delta \sin \lambda \cos ^{2} \phi\right) \\
& M_{z z}=M_{0}(\sin 2 \delta \sin \lambda) \\
& M_{x y}=M_{0}(\sin \delta \cos \lambda \cos 2 \phi+0.5 \sin 2 \delta \sin \lambda \sin 2 \phi) \\
& M_{x z}=-M_{0}(\cos \delta \cos \lambda \cos \phi+\cos 2 \delta \sin \lambda \sin \phi) \\
& M_{y z}=-M_{0}(\cos \delta \cos \lambda \sin \phi-\cos 2 \delta \sin \lambda \cos \phi)
\end{aligned}
$$

Figure 4 provide some examples of moment tensors and their corresponding focal mechanism in beachball representation.

\subsubsection{Decomposition of the seismic moment tensor}

Equation 11 relates the moment tensor components to a pure shear rupture, a DC mechanism. Such a moment tensor is characterised by eigenvalues summing up to zero and one of the eigenvalues being zero itself. The largest and smallest eigenvalue correspond to the tension and pressure axes, respectively (Eqs. 10). However, we usually have no pure DC mechanisms but seismic sources include opening/closing fractures, volume changes, or other complexities. They are connected with physical mechanisms beyond pure shear earthquakes, such as volcanic or induced seismicity. The full seismic moment tensor represents a linear combination of such effects. There are several ways to separate the different mechanisms from each other. A good overview about such 'decomposition' schemes can be found in Jost and Herrmann (1989) and Dahm and Krüger (2014).

The most common and widely used scheme is the decomposition into physical (geological) sources. These are namely the already mentioned DC part representing shear rupture, the isotropic (ISO) part representing volume changes within the source, and the compensated linear vector dipole (CLVD, Knopoff and Randall, 1970; Fitch et al., 1980):

$$
\mathbf{M}_{f u l l}=\mathbf{M}_{D C}+\mathbf{M}_{I S O}+\mathbf{M}_{C L V D}
$$

The CLVD part has no verified geological interpretation. When inverting waveforms for earthquake mechanisms, it is often interpreted as residual radiation from the DC source part. A low CLVD part is then interpreted as a confirmation of the model assumptions. However, several studies suggest that it might be more than just a mathematical leftover. Tensile faulting, where the displacement discontinuity is normal instead of parallel to the fault plane, or deep earthquakes caused by phase transformation (in subduction zones) can 


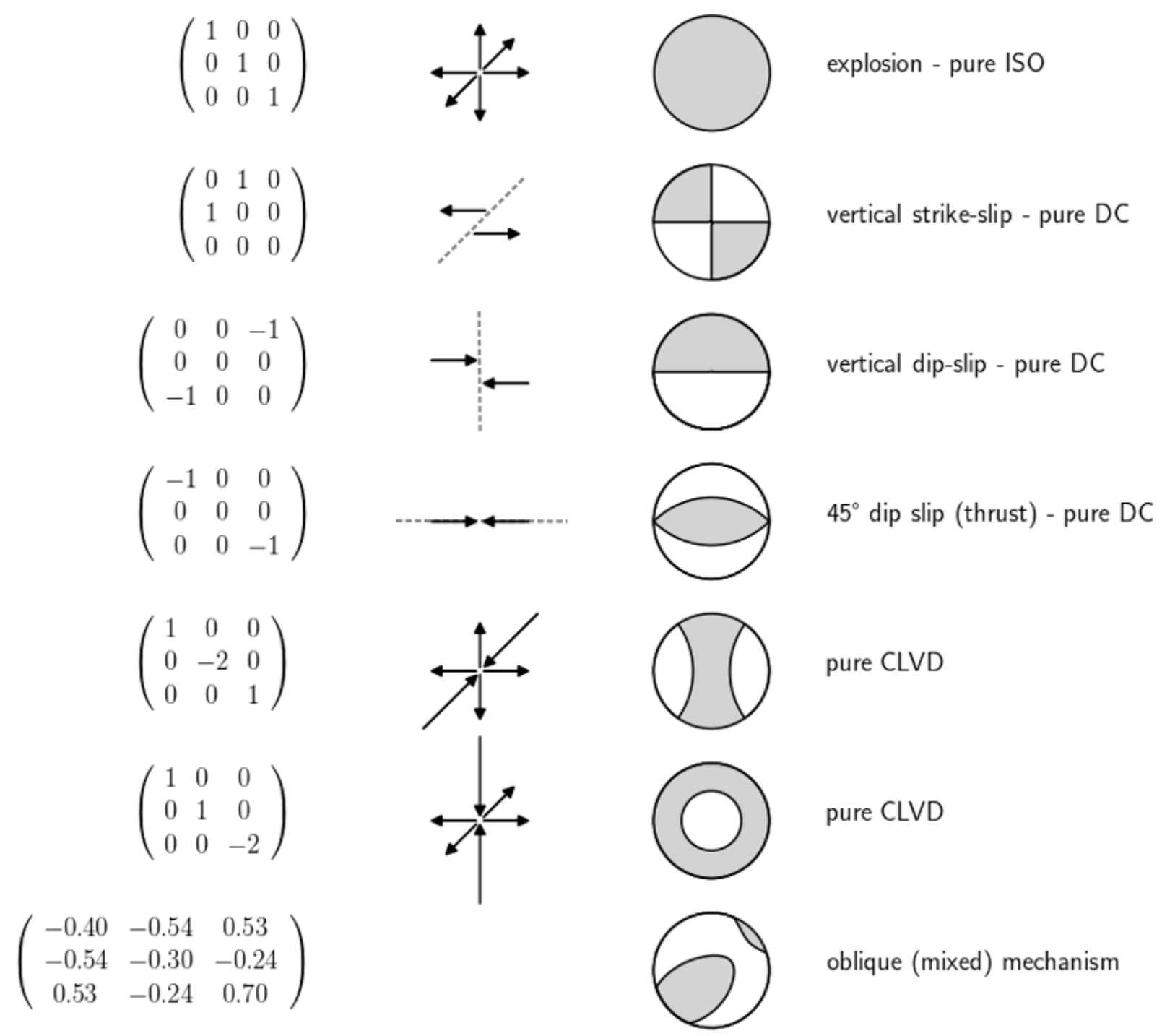

Figure 4: Examples of seismic moment tensors and their corresponding force couples and focal mechanisms. Grey dotted lines mark the axes on which the force couple acts. The arrows mark the force direction of the couple. The moment tensor components are given in the NWU (North-West-Up) coordinate system. be explained with CLVD mechanisms (e.g. Julian et al., 1998). Fault complexities such as curvatures can also cause CLVD source components (e.g. Frohlich, 1990). Furthermore, in volcano seismology a CLVD can explain ring-faulting mechanisms associated with caldera collapse (e. g. Shuler et al., 2013, Gudmundsson et al., 2016).

321 To decompose the full seismic moment tensor according to Eq. 12, it first need to be transformed into its principal axes coordinate system (see Eq. 10) by determining its eigenvalues 
$e_{i}$ and eigenvectors $\mathbf{a}_{i}$ (Jost and Herrmann, 1989):

$$
\mathbf{M}=\left(\begin{array}{lll}
\mathbf{a}_{1} & \mathbf{a}_{2} & \mathbf{a}_{3}
\end{array}\right) \mathbf{e}\left(\begin{array}{c}
\mathbf{a}_{1}^{T} \\
\mathbf{a}_{2}^{T} \\
\mathbf{a}_{3}^{T}
\end{array}\right)=\left(\begin{array}{lll}
a_{1 x} & a_{2 x} & a_{3 x} \\
a_{1 y} & a_{2 y} & a_{3 y} \\
a_{1 z} & a_{2 z} & a_{3 z}
\end{array}\right)\left(\begin{array}{ccc}
e_{1} & 0 & 0 \\
0 & e_{2} & 0 \\
0 & 0 & e_{3}
\end{array}\right)\left(\begin{array}{lll}
a_{1 x} & a_{1 y} & a_{1 z} \\
a_{2 x} & a_{2 y} & a_{2 z} \\
a_{3 x} & a_{3 y} & a_{3 z}
\end{array}\right)
$$

$$
\mathbf{M}_{I S O}=\frac{1}{3}\left(\begin{array}{ccc}
\operatorname{tr}(\mathbf{M}) & 0 & 0 \\
0 & \operatorname{tr}(\mathbf{M}) & 0 \\
0 & 0 & \operatorname{tr}(\mathbf{M})
\end{array}\right)
$$

What remains is the deviatoric moment tensor $\mathbf{M}_{D E V}=\mathbf{M}_{\text {full }}-\mathbf{M}_{I S O}$ with eigenvalues $e_{i}^{\text {dev }}$. Assuming $\left|e_{3}^{\text {dev }}\right| \geq\left|e_{2}^{d e v}\right| \geq\left|e_{1}^{\text {dev }}\right|$ the deviatoric moment tensor $\mathbf{M}_{D E V}$ can be rewritten to:

$$
\begin{aligned}
\mathbf{M}_{D E V} & =e_{3}^{\text {dev }}\left(\begin{array}{ccc}
-K & 0 & 0 \\
0 & (K-1) & 0 \\
0 & 0 & 0
\end{array}\right) \\
& =e_{3}^{\text {dev }}(1-2 K)\left(\begin{array}{ccc}
0 & 0 & 0 \\
0 & -1 & 0 \\
0 & 0 & 1
\end{array}\right)+e_{3}^{\text {dev }} K\left(\begin{array}{ccc}
-1 & 0 & 0 \\
0 & -1 & 0 \\
0 & 0 & 2
\end{array}\right)
\end{aligned}
$$

with $K=-e_{1}^{d e v} / e_{3}^{d e v}$ and $(K-1)=e_{2}^{d e v} / e_{3}^{d e v}$ and $K$ being in the range $0 \leq K \leq 0.5$. The first term on the RHS in Eq. 15 represents the DC source component while the second term stands for the CLVD part.

Visualising all source components in one plot is not a trivial task. Tape and Tape (2012) and Aso et al. (2016) provide overviews about different source-type plots. Nowadays, the most common one in use is the Hudson plot (Fig. 5, Hudson et al., 1989). It provides 'a two-dimensional graphical display of all possible relative sizes of the three principal moments' using a projection onto a cube. It has the advantage of an equal-area source-type plot. 


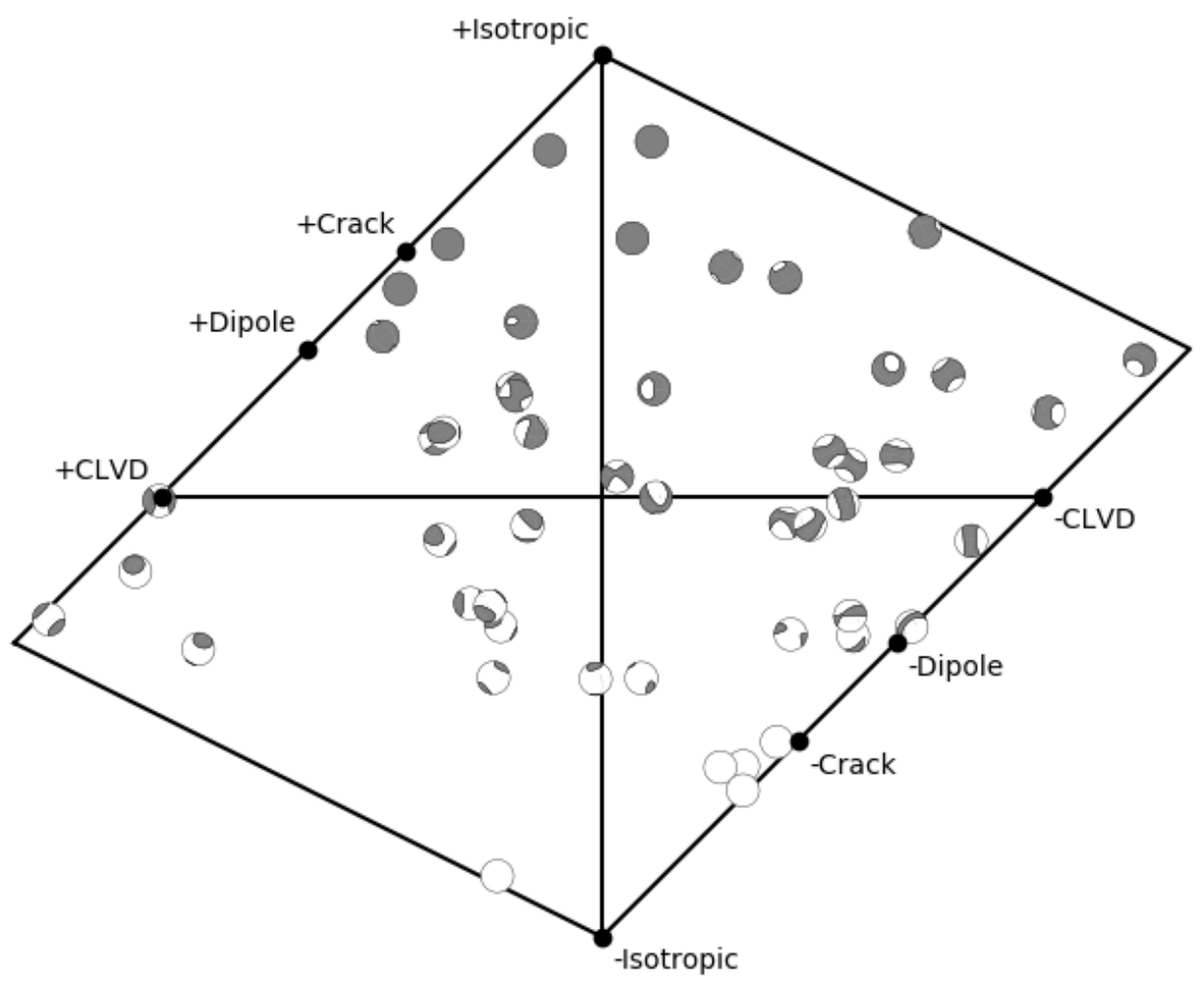

Figure 5: Source-type plot according to Hudson et al. (1989). Pure DC sources are plotted in the center spot. As an example, 50 random moment tensors are shown.

\subsection{Introduction to rotational ground motion}

\subsubsection{Fundamental theory}

The complete seismic wavefield due to a seismic source is fully described by six components (6C), three components of translational and three components of rotational ground motion; plus six components of strain when considering entire ground deformation. Rotational ground motions $\boldsymbol{\omega}$ are related to the curl of the deformation field and can be obtained through spatial derivatives of the translational wavefield $\boldsymbol{u}$ :

$$
\boldsymbol{\omega}=\frac{1}{2} \nabla \times \boldsymbol{u}=\frac{1}{2}\left(\begin{array}{l}
u_{z, y}-u_{y, z} \\
u_{x, z}-u_{z, x} \\
u_{y, x}-u_{x, y}
\end{array}\right)
$$

where $u_{i, j}$ denote the spatial derivative along the three space axes $j$. It is a vector which represents the angle of rigid rotation of the medium caused by a small deformation.

When we measure ground motion at the Earth surface, we have to consider that normal and shear traction vanish. This leads to a simplification of the equation where only horizontal 
spatial derivatives remain (Robertsson and Curtis, 2002; Cochard et al., 2006):

$$
\boldsymbol{\omega}^{F S}=\left(\begin{array}{c}
u_{z, y} \\
-u_{z, x} \\
\frac{1}{2}\left(u_{y, x}-u_{x, y}\right)
\end{array}\right) \text {. }
$$

In Eq. 17 the horizontal components are defined by spatial derivatives of the vertical translation. They are known as tilt or rocking and provide us with information on the spatial wavefield gradient of the vertical displacement with depth. This information is not available from translational point measurements at the surface. It would need large receiver arrays and data processing to access this information. The vertical component of rotational ground motion is defined by spatial derivatives of the horizontal translations and represents torsion. These relations are important to understand where the additional information with depth is coming from, mentioned later in the text several times.

In the linearised, time-domain inversion scheme for moment tensors (Eqs. 3 and 6) the ground motion components are linear combinations of the moment tensor components with the matrix of spatial derivatives of GFs. Therefore, it is straight forward to include rotational ground motion into the waveform inversion for seismic moment tensors. With the appropriate, pre-calcualed GFs simply similar linear combinations need to be set-up. The 
long version of the inversion equation based on Eq. 6 then looks like:

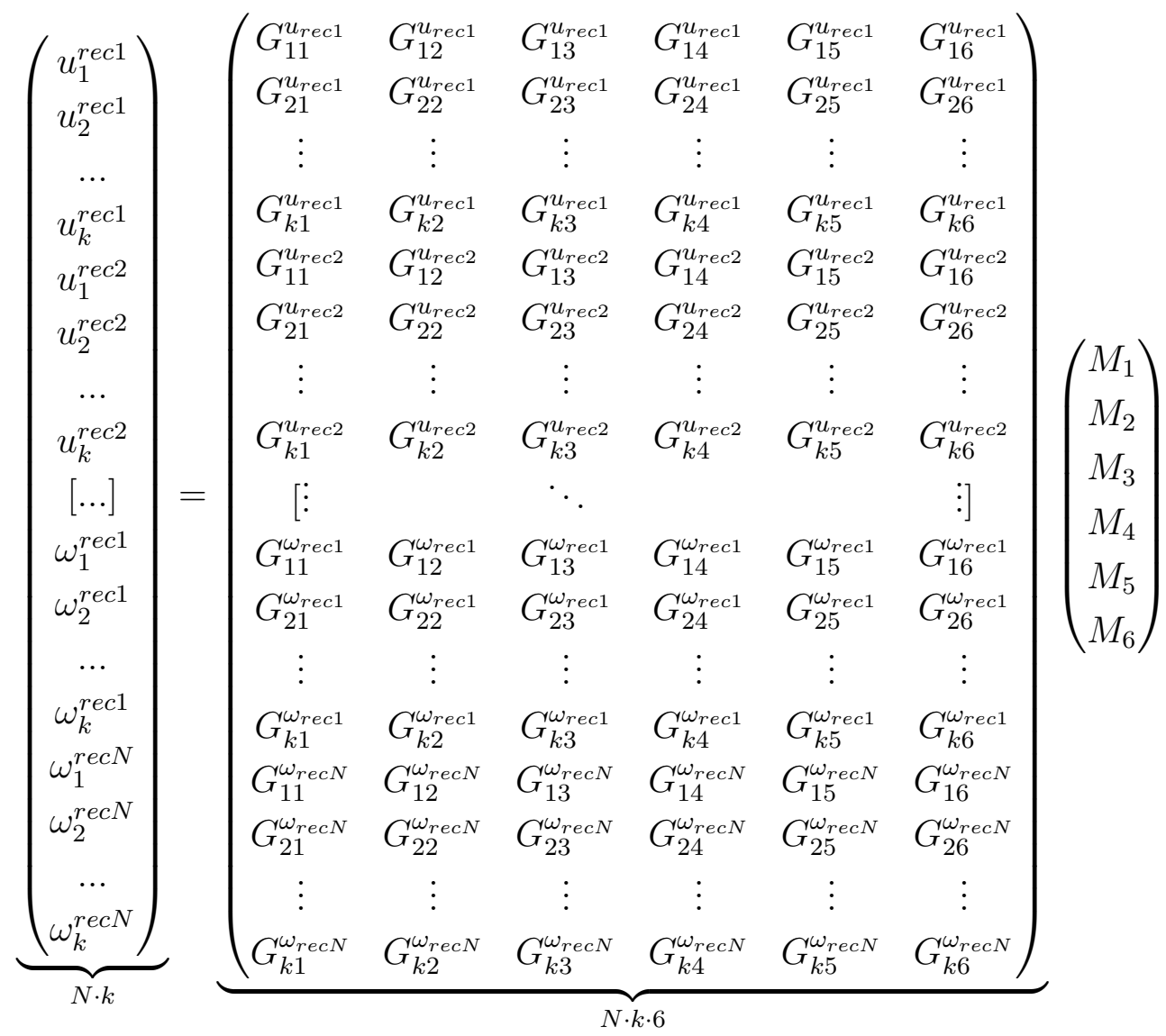

with $N$ and $k$ being the number of total recordings (translation plus rotation) and samples per recording, respectively. In Eq. $18[\ldots]$ on the LHS and [: $\ddots^{\ddots} \quad \vdots$ :] on the RHS stand for possible further entries. They are not shown to keep the equation as compact as possible. Ichinose et al. (2021) also have demonstrated this fact based on the analytical expressions for the translational and rotational ground motion components based on four fundamental source types and corresponding coefficients defined on the source-receiver azimuth and moment tensor components. The fundamental source types are vertical strike-slip, vertical dip-slip, $45^{\circ}$ dip-slip, and explosion.

\subsubsection{Measuring rotational ground motions}

Although the benefits of measuring "inclination" have been mentioned quite early (Schlüter 1903), seismology for a long time was limited to the translational part of the wavefield, with no access to the other part of the available information. Even in 2002 Aki and Richards stated: "... note the utility of measuring rotation ..., but as of this writing seismology still awaits a suitable instrument for making such measurements." This is reasoned by the very small amplitudes of rotational ground motions. Compared with translational ground motions 
they are smaller by roughly a factor of 1000, depending on the local phase velocity. Thus, for quite a while appropriate measurement facilities were missing.

A short summary about early attempts of observational rotational seismology can be found in Lee et al. (2011). Some of the first direct measurements have been done with highly sensitive ringlasers gyroscopes, an instrument originally developed for geodesy to monitor Earth's rotation (McLeod et al., 1998; Pancha et al., 2000; Igel et al., 2005; Schreiber et al. 2009). It is an interferometer, the measurement principle based on the Sagnac effect (Lefèvre, 2014). However, ringlasers are massive structures at fixed locations which need a huge effort in absolutely rigid construction to provide stable measurements. That makes them very expensive and limits the number of available measurements. Up to today only five ringlasers exist worldwide of which four measure the vertical component of rotation only.

As early as the 1990s attemps have been made to measure rotational ground motion with portable sensors (Nigbor, 1994; Takeo, 1998). Since then, several portable sensors based on very different measure principles were developed. They include the principle of torsion balance (Cowsik et al. 2009), the pairwise combination of geophones (Brokešová et al. 2012; Brokešová and Málek, 2013), liquid-based rotational motion sensors (Jedliča et al., 2012; Huang et al., 2013), magnetohydrodynamic sensors (Pierson et al., 2016), and microelectromechanical systems (MEMS)-based gyroscopes (Nigbor, 1994). Partly, these sensors were successfully used for strong motion studies (Lee et al., 2009; Nigbor et al., 2009). However, for a broad application in seismology, a higher sensitivity in a wider bandwidth is needed. A promising development with respect to that point was the usage of fibreoptic gyroscopes (Jaroszewicz et al., 2006; Velikoseltsev et al., 2012; Bernauer et al., 2021). Bernauer et al. (2018) showed that we now have the first portable rotation sensor developed for seismology which is commercially available.

According to Eqs. 16 and 17 we can derive rotational ground motions from translational surface array measurements (array-derived-rotation - ADR). The seismogeodetic method is one way to do so (Spudich et al. 1995; Bodin et al., 1997; Huang, 2003; Spudich and Fletcher, 2008, 2009). Here, the best-fitting spatially uniform strain and rigid body rotation is determined to fit the instantaneous deformation of an array of seismic recorders and, thus, provides a spatial average of rotational ground motions over the area of the surface array. Pand S-wave velocities beneath the array must be known and the method assumes that the deformation is linear over the array area. Therefore, the validity of the resulting rotational ground motion is limited to lower frequencies, depending on the spatial extend of the array. Suryanto et al. (2006) also stated, that although the method needs measurements of only three stations, the accuracy of the derived rotation is considerably influenced by noise within the translational data. However, the more recordings are used to derive rotational ground motions, the better this influence is cancelled out. Other factors influencing the accuracy 
of derived rotational ground motions are topography, structural heterogeneities, receiver location uncertainties, uncertainties in the seismometer response function, strain-rotationcoupling, and inaccuracies in the plane wave assumption. Nevertheless, comparisons between ADR and direct measurements showed excellent fit between both with correlation coefficients up to $98 \%$ (Suryanto et al., 2006; Wassermann et al., 2009; Lin et al., 2012; Donner et al. 2017). Therefore, it should be possible to use them as an addendum to direct measurements for waveform inversion. The method of Spudich and Fletcher (2008) is implemented in the Python-based seismological analysis toolbox ObsPy (Beyreuther et al., 2010; Megies et al. 2011; Krischer et al., 2015).

Langston (2007a b c, 2018) developed a very similar method complemented by a weighting scheme and coined the term 'wave gradiometry' for it. Instead on the resulting arrayderived rotation and strain, he rather focuses on determining the wavefield gradient with high precision (Liang and Langston, 2009).

\subsubsection{Rotations due to a double-couple point source}

Applying the same notation as for Eq. 9 and given in Fig. 3 for the translational rotation pattern, we can find a likewise expression for the rotational radiation pattern (Cochard et al., 2006; Igel et al., 2015):

$$
\boldsymbol{A}^{R}=\cos \theta \sin \phi \hat{\boldsymbol{\theta}}+\cos \phi \cos 2 \theta \hat{\boldsymbol{\phi}}
$$

Another way to obtain the rotational radiation pattern is to calculate the curl of the transverse far-field radiation pattern (Eq. 9, Cochard et al., 2006). That is $\nabla \times \boldsymbol{A}^{F S}=\boldsymbol{A}^{R} / r$. Hence, it becomes clear, that these two radiation patterns are orthogonal to each other (see also Sec. 3.2).

\subsubsection{Strain-rotation coupling and local side effects}

Similarly to translational ground motions, direct measurements of rotational ground motions are biased due to local side effects. Small-scale heterogeneities and rough topography, for example, can cause a conversion of strain into rotation (strain-rotation-coupling or straininduced rotation, King and Bilham, 1973; Harrison, 1976; Kohl and Levine, 1995; van Driel et al., 2012, 2015). This effect can significantly contribute to the measurement of rotational ground motion on all distances and in the low-frequency spectrum (van Driel et al., 2012). The same authors also found a dependence on the relative magnitude of strain and rotation. In turn, the relative magnitude of strain and rotation is influenced by the radiation pattern, source-receiver-distance, or seismic phases.

The effects of local side conditions, that means small scale heterogeneities of the structure, 
on rotational ground motion is more significant than for translational data. For example, Fichtner and Igel (2009) and Bernauer et al. (2012) could show that the finite-frequency sensitivity kernels of rotational ground motion are influenced only by structure in close vicinity of the receiver but not along the path or in the vicinity of the source. Thus, they could be used for single receiver local tomography.

These findings have important implications when working with direct measurements of rotational ground motion. Under specific circumstances the data are usable only when they are corrected for local side effects, especially strain-rotation-coupling. (Singh et al., 2020) investigated the coupling in more detail using the theory of homogenisation (Capdeville et al. 2020). They introduced the coupling vector $\boldsymbol{J}$ which is a characteristic of the receiver location and not dependent on source or time. Therefore, it is possible to invert for the coupling vector $\boldsymbol{J}$ and subsequently use it to correct the measured data of a specific receiver location.

\section{Benefits of rotational ground motions for regional seismic moment tensors}

When including recordings of rotational ground motions into the waveform inversion for seismic moment tensors, we extend the number of observations of the seismic wavefield. Intuitively, we understand this addition in information alone should improve the resulting moment tensor solution. Because portable rotational sensors are quite new instruments, so far, there are no studies based on real, direct measurements of rotational ground motion. The studies summarised in this review worked with either synthetic waveforms or with ADR measurements.

This section is structured as follows: At first, some general benefits are presented (section 3.1) before the influences of receiver number and geometry are explained (section 3.2). Section 3.3 discusses the influence of structural model and its relation to inverted frequencies as well as the relation between frequencies, magnitudes, distance ranges and used waveform types. Section 3.4 focuses on the resolvability of centroid depth. A discussion on the resolvability of different source parts can be found in section 3.5 before findings on the influence of noise are presented in section 3.6 .

In the following, the term 'resolution' is used to describe the quality, or reliability, of the resulting moment tensor from waveform inversion. In this review, the term refers to both, the accuracy and the precision with which the moment tensor components are determined. 


\subsection{General benefits for the resolution of the moment tensor com- ponents}

Donner et al. (2016) investigated the waveform inversion results from six component (6C, translation+rotation) synthetic waveforms in a scenario based on a 1-dimensional (1D) structural model of northern Iran. The synthetic waveforms are calculated based on a synthetic shallow strike-slip source in $6 \mathrm{~km}$ depth with a moment magnitude of $\mathrm{M}_{w} 4.0$ and with $80 \%$ DC. The seismograms were calculated up to $0.1 \mathrm{~Hz}$ with a minimum wavelength of $27 \mathrm{~km}$. Therefore, the dominant wave type are surface waves as it is standard in regional waveform inversion for moment tensors. For the receiver locations the authors chose two scenarios: one based on the real distribution of broadband stations of the Iranian National Seismic Network (INSN) operated by the Institute of Earthquake Engineering and Seismology, and a second scenario based on a regular grid of virtual receivers covering almost entire Iran (Fig. 6). To obtain an unbiased solution selection and also consider possible trade-offs the authors performed a Bayesian (probabilistic) inversion based on equations from Tarantola (2005). A prior probability density function $(\rho(\mathbf{m})$, prior pdf) on the parameter space $\mathbf{m}$ (moment tensor components and centroid depth) is connected with a Likelihood function to obtain the posterior probability density function $(\sigma(\mathbf{m})$, posterior $p d f)$. The likelihood function provides a measure of how well a model is fitting the data and is basically defined as the L2 norm between the synthetic and theoretical observations. The parameter space is randomly sampled using the Metropolis-Hastings algorithm testing one million start solutions (Metropolis and Ulam, 1949; Metropolis et al., 1953; Hastings, 1970). The posterior $p d f$ is quantified relative to the prior pdf using Shannon's measure of information gain (IG, Shannon, 1948):

$$
I G(\rho, \sigma)=\int \rho(x) \log \left[\frac{\rho(x)}{\sigma(x)}\right] d x .
$$

The unit of the information gain is termed a bit due to the logarithm base 2 .

In their first scenario, Donner et al. (2016) compared the three component (3C) versus six component (6C) inversion results based on two rings of virtual recorders distributed around the source. To keep the amount of data constant during inversion, they halved the number of recorders when including the rotational data to the inversion (black dots with white crosses in Fig. 6a). Hence, the improvement of the result does not come from purely increasing the amount of data but actually from adding new information. In this case, the IG increased by $53 \%\left(\left(\mathrm{IG}_{6 C}-\mathrm{IG}_{3 C}\right) / \mathrm{IG}_{3 C} \times 100 \%\right)$. Especially, components including spatial derivatives with respect to depth are significantly better resolved. For component $\mathrm{M}_{z z}$ the IG increased by $161 \%$. The same comparison based on the real station distribution (second scenario of this study) confirmed the findings with an overall increase of IG of $136 \%$. That means, 


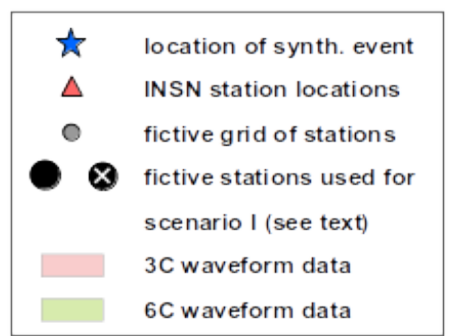

b)
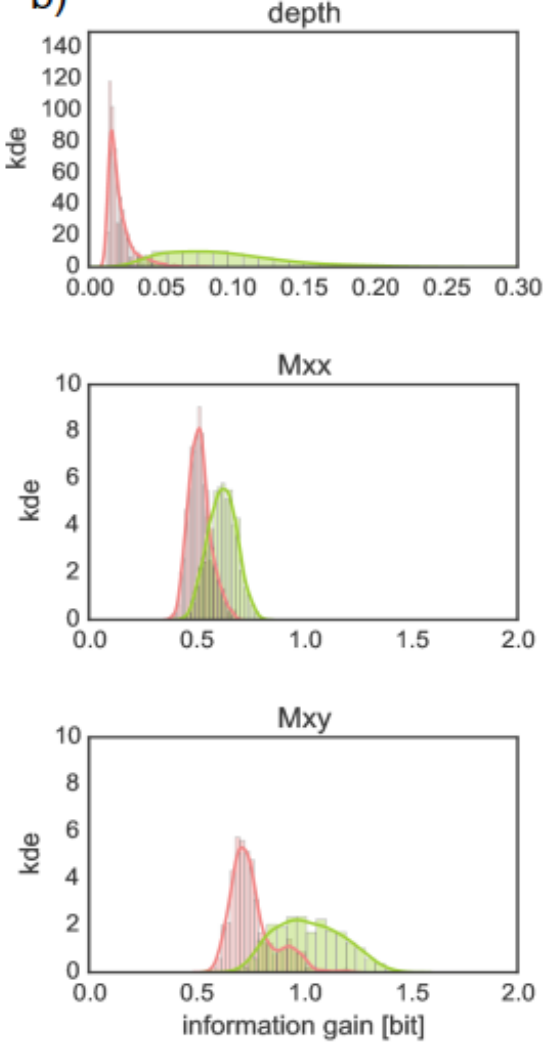
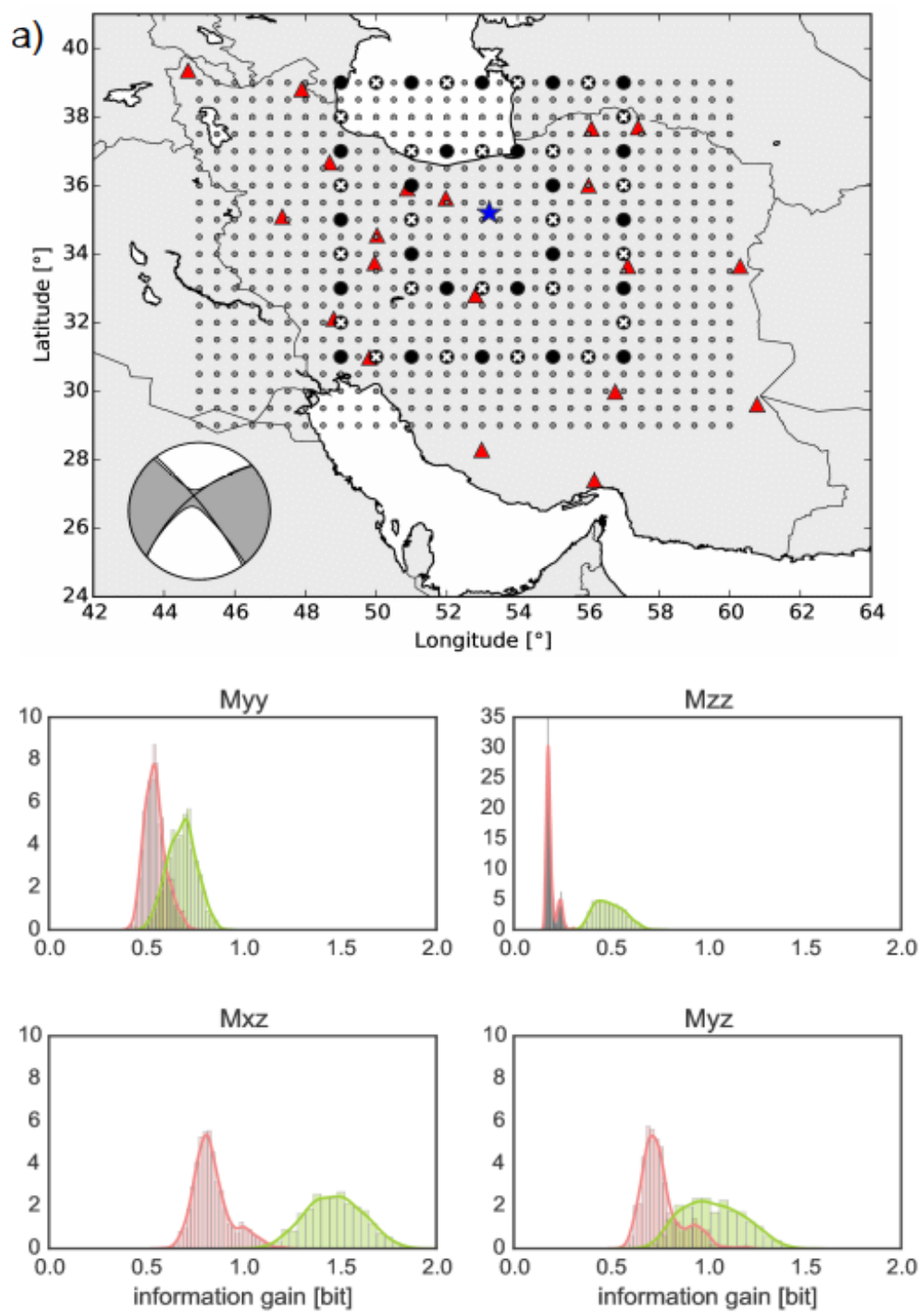

Figure 6: Summarised study results of Donner et al. (2016, 2018). a) Overview map for the study area. Blue star shows the location of a synthetic source with its mechanism given by the beachball (grey: full moment tensor; black: DC part). Red triangles mark the real station locations of the Iranian National Seismic Network (INSN) while dots represent a virtual grid of receivers. Black and white-crossed dots (6C and $3 \mathrm{C}$ receivers, respectively) are the receivers used in their scenario one. b) Gaussian kernel density estimations for the IG corresponding to 1000 runs based on the virtual grid of receivers. Coral and green distributions show the IGs from inverting $3 \mathrm{C}$ and $6 \mathrm{C}$ waveform data from randomly chosen receivers for the given parameters, respectively. The higher the IG, the more the inverted parameter benefits from using rotational motions. Same IG means same inversion resolution using half the number of receivers but the same amount of data. (Modified after Figs. 1 and 3 of Donner et al. (2016).) 
with half the number of receivers the resulting moment tensor is better resolved using $6 \mathrm{C}$ compared with only $3 \mathrm{C}$ waveform data. Most probably, the benefits come from the horizontal components of the rotation (tilt) holding information on the vertical displacement gradient (see also section 2.2.1).

This assumption could be confirmed by a study on the kinematic source solution of a buried fault (Reinwald et al, 2016). Here, the authors applied the same Bayesian inversion scheme inverting for slip on the rupture plane, rupture velocity and rise time. They compared the inversion results for a strike-slip and a dip-slip source with exactly the same synthetic setup and source-receiver-geometry for both synthetic sources. For the strike-slip source the increase in IG due to adding rotational recordings to the inversion was in total $10 \%$ while for the dip-slip source the increase in IG was $32 \%$. Especially, the resolution of the rupture velocity, rise time, and the slip closest to the Earth surface benefited significantly from the rotational ground motions. In addition, already Bernauer et al. (2014) could show that the well-known trade-off between rupture velocity and rise time can be reduced substantially. Applying the same inversion scheme, the authors tested a synthetic strike-slip source only. They showed the 2D marginal posterior pdf of rupture velocity and rise time for the $3 \mathrm{C}$ and $6 \mathrm{C}$ scenario. The area where variations in both parameters compensate each other is about twice as large for the $3 \mathrm{C}$ case than for the $6 \mathrm{C}$ case. Also, in the $3 \mathrm{C}$ case the expected values from the posterior $p d f$ missed the target value considerably, the inaccuracy being larger for the rise-time than for the rupture velocity. For the $6 \mathrm{C}$ case, the increase in $\mathrm{IG}$ for rupture velocity and rise time was $25 \%$ and $60 \%$, respectively.

A study with real, ADR-based measurements was performed by Ichinose et al. (2021). They used translational measurements from the Piñon Flat Observatory Array and the Golay Array in the US to derive rotational ground motion (see Sec. 2.2.2). The authors performed a waveform inversion for the full moment tensor (long period, time-domain, and linear inversion) and a Network Sensitivity Solution (NSS) analysis (Ford et al., 2010) to investigate the resolution of the resulting moment tensors. In addition, they intentionally reduced the number of measurements for inversion to three receivers because they argue that adding more data to an already large dataset would not necessarily improve the solution (without applying weighting schemes). Ichinose et al. (2021) found similar improvement for the moment tensor resolution as Donner et al. (2016, 2017) which manifest in a reduction of the non-DC components (as discussed in Sec. 3.5) and a decrease of the area of certain percentage of variance reduction level in the NSS analysis.

Most generally, we know that some moment tensor components are very difficult to resolve from translational ground motion in the regional distance range. Some specific source characteristic cannot be determined from Love or Rayleigh surface waves alone (for a discussion on body versus surface waves see section 3.3. For example, the component $\mathrm{M}_{z z}$ can only 
be determined in combination with $\mathrm{M}_{x x}$ and $\mathrm{M}_{y y}$ from Rayleigh waves alone, affecting the resolution of the ISO source part (Julian et al., 1998). Furthermore, because for shallow sources some moment tensor components $\left(\mathrm{M}_{x z}, \mathrm{M}_{y z}\right)$ do not contribute to the surface wave radiation pattern due to the free surface condition (Bukchin, 2006; Bukchin et al., 2010). Yet, due to their dependence on the vertical displacement gradient, these components are also the ones benefiting most from including rotational ground motion into the waveform inversion.

\subsection{Influence of the receiver distribution}

Fig. 6b summarises the generalised inversion results based on the virtual grid of receivers from Donner et al. (2016). They repeated the Bayesian inversion a 1000 times randomly selecting receivers out of the regular grid to further investigate the effect of the receiver distribution. Fig. 6b shows the Gaussian kernel density estimation ( $k d e$ ) over the IG from these 1000 inversions with one million start solutions each for the seven parameters inverted for. That means, the further the distribution stretches to the right side of the panels, the better is the parameter resolved. The coral and green distributions correspond to inversion results of $3 \mathrm{C}$ and $6 \mathrm{C}$ waveform data, respectively. Again, for the $6 \mathrm{C}$ case only half the number of receivers but the same amount of data was inverted. For the moment tensor components $\mathrm{M}_{z z}$ and $\mathrm{M}_{x z}$ it shows, no matter how the receivers are distributed around the source, the parameters are always better resolved when including rotations into the inversion. For the other moment tensor components it depends on the receiver distribution whether the component is equally well or better resolved. In Fig. 6b, these two cases correspond to both distributions partially overlapping or the $6 \mathrm{C}$ distribution being shifted to the right to higher IG, respectively.

Theoretically, it should be possible to determine the full seismic moment tensor from a single $3 \mathrm{C}$ recording when all seismic wave types are recorded because in the time domain it is an overdetermined inversion problem (see Eqs. 3 and 6). Ekström et al. (1986) has shown it for the teleseismic distance range. However, in practise the attempt is rarely successful in the regional distance range, mainly due to more complex waveforms and, hence, a higher necessity for detailed structural models compared with the teleseismic distance range (see also section 3.3). Therefore, often only a constrained moment tensor or even just the DC part can be obtained from a single 3C recording. Donner et al. (2018) have analysed this issue including rotational ground motions using the same inversion scheme and set-up as in their 2016 study (see Fig. 6). They investigated the outcome from one, two, and three receiver locations. They conclude that the probability to retrieve a reliable full moment tensor solution from the $6 \mathrm{C}$ recordings of only two or three receivers is very high. The increase in IG compared with inverting 3C recordings only was $178 \%$ and $181 \%$, respectively. However, 
from only one $6 \mathrm{C}$ recording alone the full moment tensor is probably still not retrievable in many cases. The possibility to resolve a constrained moment tensor, for example the DEV moment tensor or only DC part, was not tested but the authors argue that it might be possible. The IG from inverting a single $6 \mathrm{C}$ recording for the full moment tensor increases by $105 \%$ compared with inverting a single $3 \mathrm{C}$ recording.

In their study based on ADR data, Ichinose et al. (2021) inverted data of three receivers, of which only one is a $6 \mathrm{C}$ receiver, for the full moment tensor. The authors demonstrated a significant increases in the DC part of the source mechanisms compared to inverting translational recordings alone.

In the study of Donner et al. (2018), a second reason for the improved resolution of the moment tensor due to including rotations into the inversion could be identified. Fig. 7 shows the radiation pattern for the theoretical earthquake source used in the mentioned studies, that means the normalised maximum energy for each receiver of the virtual grid. It is calculated as the square root of the sum of the squared amplitudes divided by the maximum amplitude of the trace: $E=\sqrt{\sum t r_{i}^{2}} / A_{\max }(t r)$. It is clearly visible that an optimum choice of receiver location within this radiation pattern provides strongly varying amplitude ratios, constraining the waveform inversion for the seismic moment tensor effectively. From the three components of translational ground motion alone only three amplitude ratios are possible. When including the rotational ground motion this number increases to 15 amplitude ratios providing significantly more constraint for the inversion (enumerative combinatorics: two out of six without multiple selection and without considering order). Of course, we can perform this analysis only after the earthquake occurred and then it is hardly possible anymore to decide for the perfect receiver location for this specific earthquake. However, seismological observatories usually have a good overview of their monitored region and know where to expect seismic activity and what general kind of earthquake mechanisms, at least approximately. In addition, in some settings we know quite well which kind of seismicity to expect at which location, for example, when monitoring geothermal facilities, mining, or volcanic areas. Hence, for some cases it is possible to roughly determine which receiver location would be more optimal than others in advance.

This argumentation was also demonstrated by Ichinose et al. (2021) using a finite difference simulation of the curl and displacement wavefields for regional distance receivers and arguing on the orthogonality of the rotational and far-field SH radiation patterns (see Sec. 2.2.3 and Cochard et al., 2006). They summarised the relation as follows: 'One 6-C sensor gathers the same information on long-period radiation pattern as two 3-C at $90^{\circ}$ azimuth from one another on the focal plane'. Fig. 8 demonstrates the effect with synthetic, noise-free waveforms of a dip-slip event (strike $=45^{\circ}$, dip $=50^{\circ}$, and rake $=-70^{\circ}$ ) in a depth of $6 \mathrm{~km}$ based on a local 1D structural model. The theoretical receivers are located in the same distance 

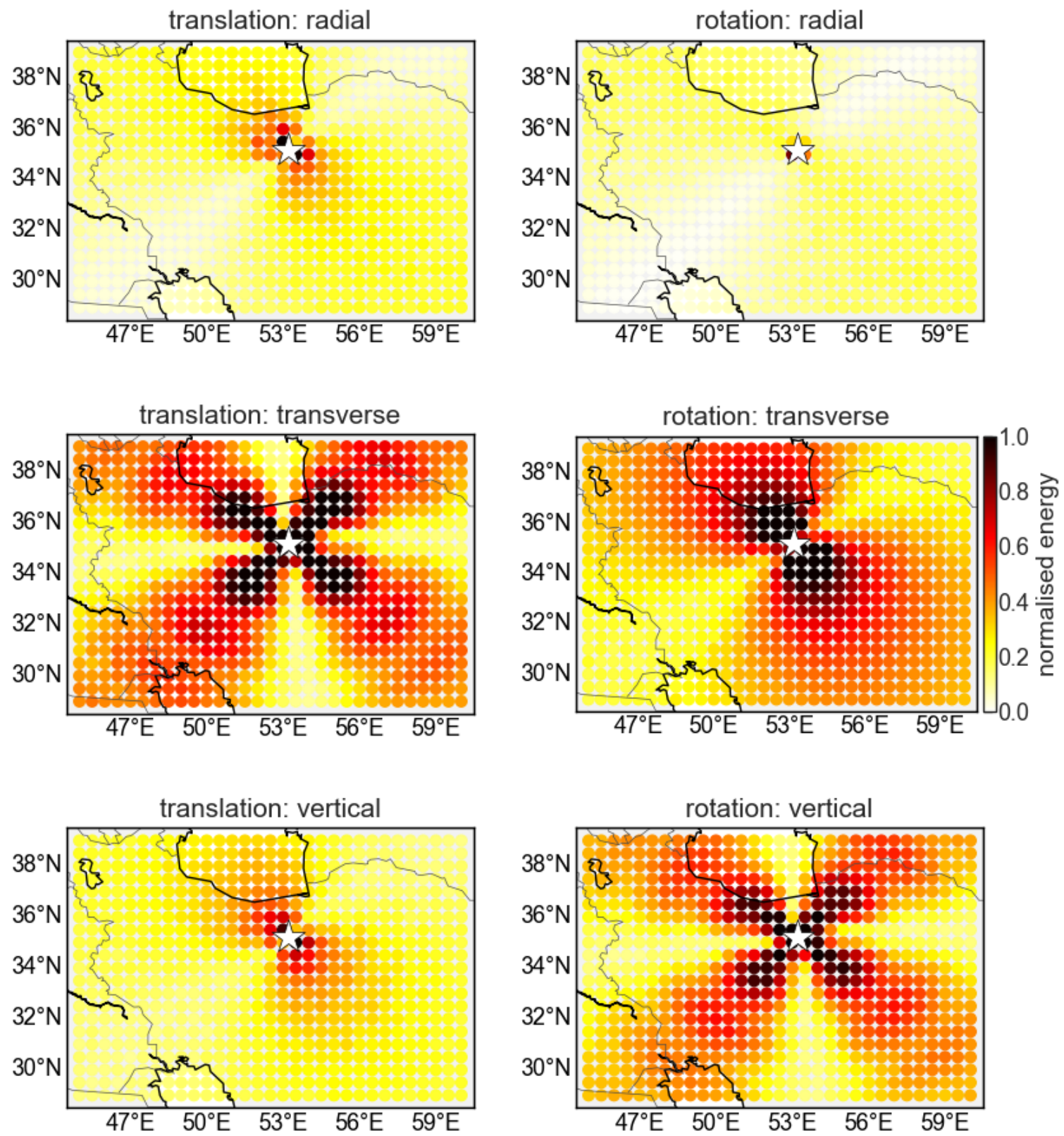

Figure 7: Radiation pattern for the synthetic earthquake scenario in Fig. 6. Upper and lower row show the normalised energy amplitudes for translation and rotation while from left to right the radial, transverse and vertical components are shown, respectively. White star marks the epicenter of the theoretical source. Modified after Fig. 8 of Donner et al. (2018). 
with varying azimuths in steps of $30^{\circ}$ around the source. The waveforms are normalised to one over all components, evaluating translations and rotations separately because of their large amplitude difference. The dominant wave is the $\mathrm{S}$-wave, the $\mathrm{P}$-wave is only lightly visible on the vertical translation data. The increase/decrease of the amplitudes with increasing azimuth clearly show the difference in azimuth for the maximum amplitudes between both types of waveform data. This information leads to meaningful amplitude ratios which are exploited during waveform inversion.
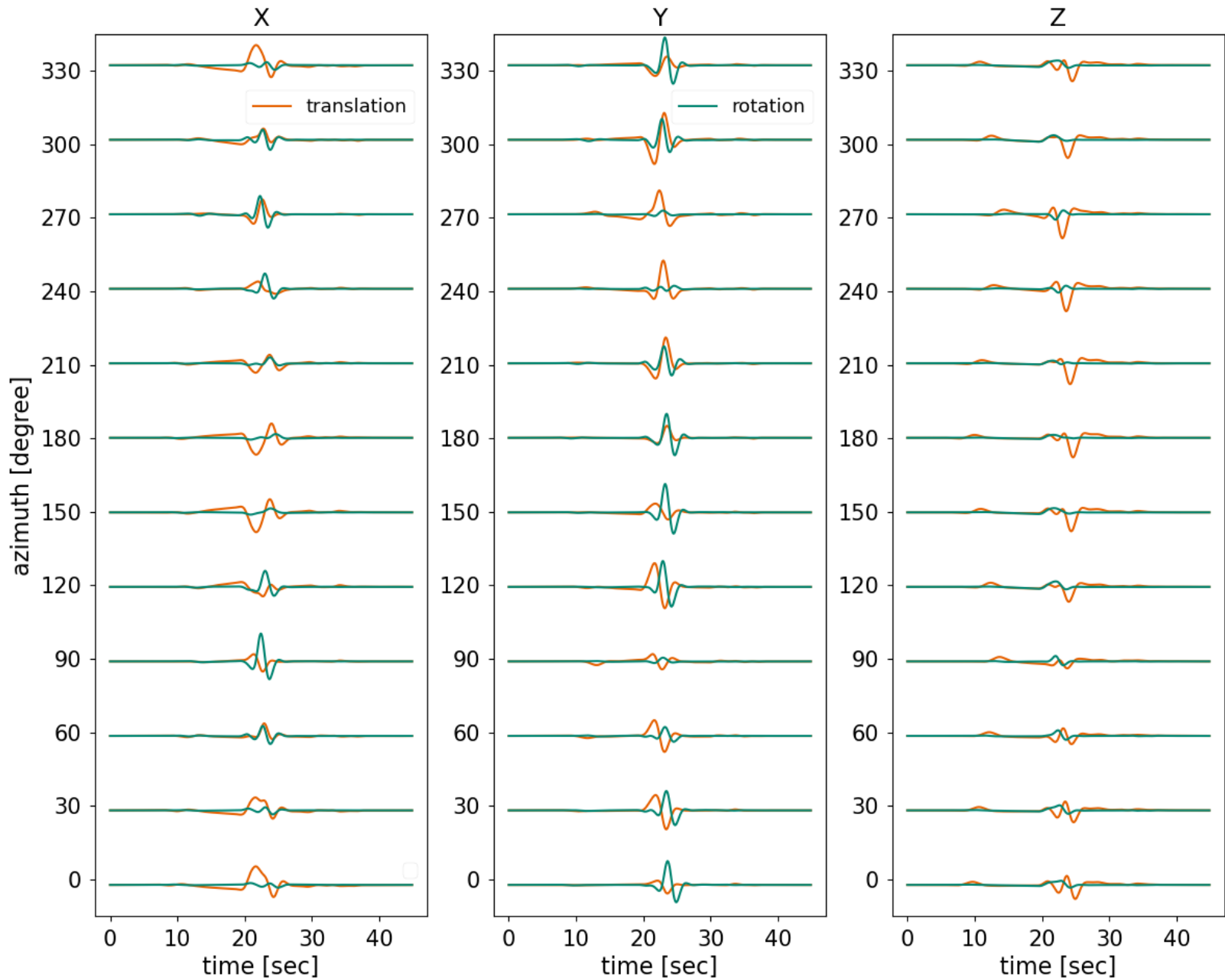

Figure 8: Synthetic waveforms for a dip-slip event (strike $=45^{\circ}, \operatorname{dip}=50^{\circ}$, and rake $=-70^{\circ}$ ) with $6 \mathrm{~km}$ depth recorded at the same distance but with different azimuths in steps of $30^{\circ}$. Amplitudes are normalised to one, evaluating translations (orange) and rotations (turquoise) separately. The dominant amplitudes are the S-wave. The varying amplitudes over azimuth clearly show the orthogonality of the radiation pattern between translations and rotations. 


\subsection{Influence of frequency range and structural model}

In the regional distance range, it is standard to invert the waveforms in a frequency range appropriate to cover the surface waves, which is roughly between 0.01 and $0.1 \mathrm{~Hz}(10-100 \mathrm{sec})$ with a focus between 0.02 and $0.05 \mathrm{~Hz}(20-50 \mathrm{sec})$. In this frequency range it is the surface waves carrying most of the energy and, thus, yielding a lot of information into the inversion. The choice of frequencies is also related to the magnitudes of the analysed events. The lower the magnitude, the higher is the corner frequency of the event and the radiated frequency content (Fig. 9). In the regional distance range, we usually analyse earthquakes with a magnitude roughly between $\mathrm{Mw} 4.0$ and $\mathrm{Mw}$ 6.5. For these events, the frequency content of the radiated surface waves is safely below the corner frequency of the events. Above the corner frequency the simplifying assumption of a point source (see Sec. 2.1.1) and thus the mathematical equation for the waveform inversion is no longer valid. Below and above these approximate magnitude thresholds, we rather speak of local and teleseismic waveform inversion, respectively. Though, the boundaries between these categories are not defined precisely.

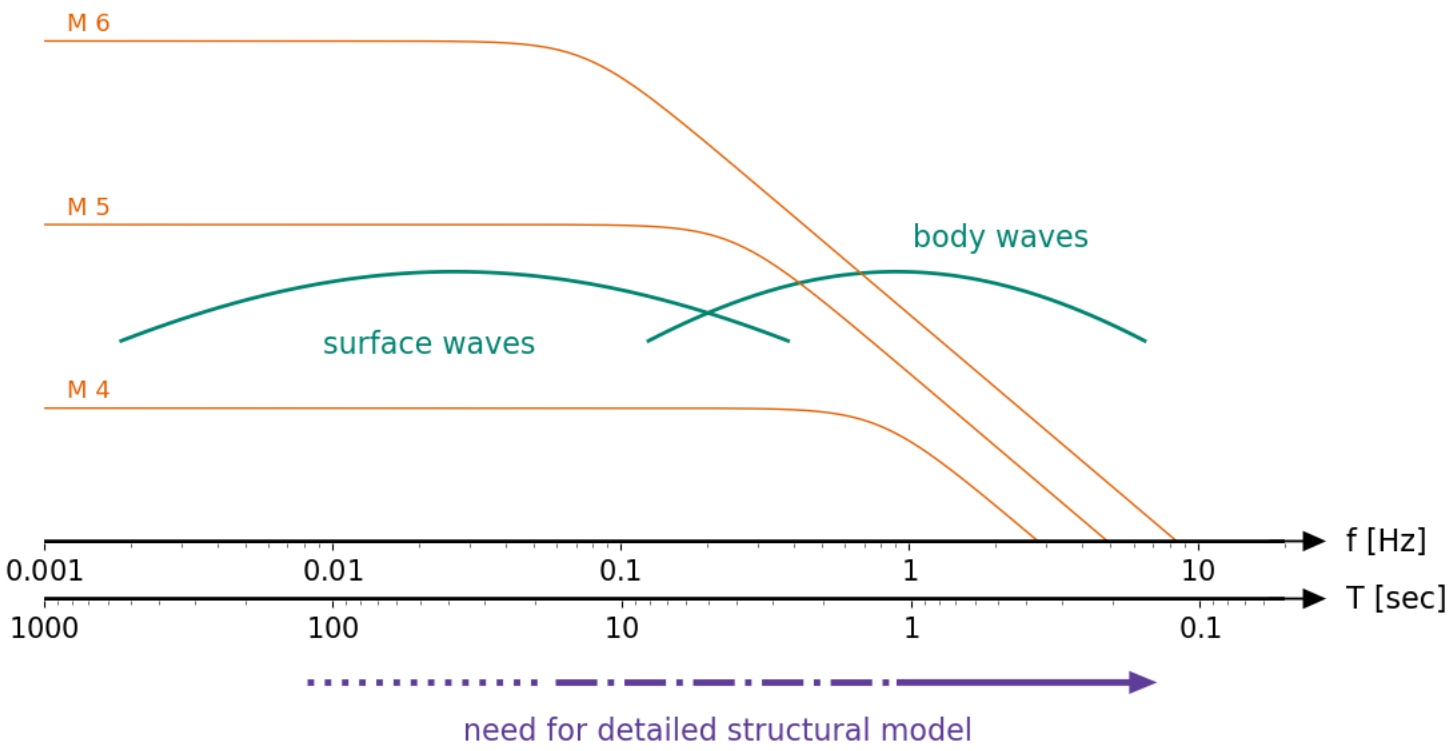

Figure 9: Schematic representation of the relationships between earthquake magnitude and corresponding corner frequency (orange), seismic wave type (turquoise), and structural model accuracy (purple) with respect to frequency and period range. The given values represent approximate guidelines and no exact limits.

The choice of frequencies is also connected to the accuracy of the structural model on which the calculation of the GFs for the inversion is based. Low frequencies are related to rather long wavelengths. In the case of surface waves the wavelength is several tens of kilometres as 
a minimum. As a consequence, the seismic wave is 'blind' for smaller details of the underlying structure. Very often, a 1D structural model, where seismic properties vary only with depth but not laterally, is sufficient to reliably invert for seismic moment tensors in the regional distance range. According to Šilený (2004) for the inversion in the frequency range 0.02 $0.05 \mathrm{~Hz}$ an uncertainty of the crustal structure up to $30 \%$ is harmless before it starts to bias the inversion result for the event-receiver geometry he tested. Also Vasyura-Bathke et al. (2021) concluded that uncertainties in the structural model can affect the moment tensor resolution profoundly. Especially, the resulting decomposition suffered from structural model errors.

For a lot of regions such 1D structural models meanwhile exist, for example, from local earthquake tomography (e. g. Eberhart-Phillips, 1990; Thurber, 1993; Haberland et al., 2009; Mousavi et al. 2015). However, seismic waveforms are influenced the most from the upper crust, which is the most complex part of the Earth's structure and includes strong lateral heterogeneities on all spatial scales. A structural model perfectly describing arrival times of seismic body wave phases is not necessarily sufficient for surface wave waveform inversion but in the best case can be adapted (e. g. Donner et al., 2013).

Since a couple of years, the progress in computational and storage resources allowed to investigate the usage of 3-dimensional (3D) structural models for various studies, including regional waveform inversion for moment tensors (e.g. Hingee et al., 2011; Zhou et al., 2016; Hejrani et al., 2017). Several authors have shown that the usage of 3D structural models can be strongly beneficial for the reliability of the inversion result, that means the seismic moment tensor (e.g. Fichtner and Tkalčic, 2010; Kim et al., 2011; Covellone and Savage, 2012; Kühn and Vavryčuk, 2013; Hejrani and Tkalčić, 2020). It allows to extend the waveform inversion to higher frequency ranges, but still below the corner frequency, because the chances to decipher the more complex waveforms due to more detailed structure increase. This instance shifts the focus from surface waves to include body wave energy adding physical information to the inversion and, thus, can improve the resolution of the resulting moment tensor. It also allows to lower the magnitude threshold for which waveform inversion for seismic moment tensors are possible because smaller events have a higher corner frequency.

Fig. 9 suggests that the radiated energy of seismic events is constant and easy to use at the lower end of the frequencies but this is not entirely true. Real world observations show that the plateau of the displacement amplitude spectrum does not spread endlessly to lower frequencies but rises again below a certain frequency. This is due to the influence of noise superimposing the earthquake signal. Thus, it is difficult to resolve the moment tensor of small events from low frequencies and more detailed models are needed to fit the higher frequencies. Nevertheless, 3D structural models and GFs calculated on them need to be carefully validated against the true structure. If they do not represent the real conditions 
properly, they rather harm the inversion result than improve it (e.g. Graves and Wald, 2001; Hjörleifsdóttir and Ekström, 2010).

These relationships have long been known from the inversion of translational ground motions. They are still just as valid when including the rotational ground motions into the waveform inversion. The question remains what factor improves the reliability of the resulting moment tensor more: inverting 6C waveforms or GFs based on 3D structural models? Donner et al. (2020) tried to answer this question, again based on the same Bayesian inversion scheme as before comparing $3 \mathrm{C}$ versus $6 \mathrm{C}$ waveform inversion. This time, the set-up is a scenario on the Korean Peninsula with synthetic waveforms based on a shallow strike-slip Mw 5.4 earthquake in the Republic of Korea (ROK) and based on an Mw 5.8 explosive source in the Democratic People's Republic of Korea (DPRK). They calculated two sets of GFs based on a 1D and a 3D structural model and compared the inversion results for both sets in three different frequency ranges: $0.02-0.05 \mathrm{~Hz}(20-50 \mathrm{sec}), 0.02-0.1 \mathrm{~Hz}(10-50 \mathrm{sec})$, and $0.02-0.16 \mathrm{~Hz}(6-$ $50 \mathrm{sec}$ ). Their main conclusion is that for both event scenarios the combination of $6 \mathrm{C}$ with $3 \mathrm{D}$ improves the reliability of the result the most. This effect can even be increased when broadening the frequency range to higher frequencies.

However, they found differences for the two source types. For the explosive event the usage of a 3D model turned out to be superior than adding the rotational ground motion to the inversion, especially in the higher frequency range. This is explained by the type of radiated energy. Explosive sources radiate most of their energy as compressional ( $\mathrm{P}-)$ wave energy and only very few shear-wave energy, i. e. S- and surface wave energy. They contain much higher frequencies than surface waves, which strengthens the preference for 3D instead of $6 \mathrm{C}$ for explosive sources. In addition, compressional waves cannot excite rotations in a homogeneous medium and considering the far-field energy radiation. Though, Pham et al. (2010) showed with real data examples that we do see rotations in the P-wave train. It is assumed that scattering and P-to-S conversion due to anisotropy in the upper crust is responsible for the effect. As a consequence, the rotational ground motions indeed do add physical information to the waveform inversion of explosive sources but being able to model crustal details and invert higher frequencies is more relevant here.

Finally, as already mentioned in Sec. 2.2, the distortions of the recorded waveforms due to local site effects are more severe for rotational than for translational ground motions (though not negligible here as well). Most likely it will not be possible to invert real, direct recordings of rotational ground motion without carefully considering the coupling vector $\boldsymbol{J}$. The verification is pending. 


\subsection{Resolution of the centroid depth}

For a long time, it is well know that the focus on surface wave frequencies in the regional distance range comes with a major draw back. The equations for the radiation patterns of Love and Rayleigh surface waves contain terms including spatial derivatives of the eigenfunctions with respect to depth. The terms in question affect the moment tensor components $\mathrm{M}_{x z}$ and $\mathrm{M}_{y z}$. They are proportional to the shear traction on a horizontal plane. At the free surface, the shear traction vanishes. As a consequence, the connected moment tensor components are badly resolved for shallow sources (centroid depth « considered wavelength, Dufumier and Cara, 1995; Bukchin, 2006; Bukchin et al., 2010).

In their second scenario based on the real station distribution of the INSN (red triangles in Fig. 6a) Donner et al. (2016) found a bimodal distribution for the $k d e$ of the centroid depth for both inversions, based on $3 \mathrm{C}$ and $6 \mathrm{C}$ waveform data; one for a shallow depth of $4-8 \mathrm{~km}$ and one for a deeper depth of $16-20 \mathrm{~km}$ (target centroid depth: $6 \mathrm{~km}$ ). The $3 \mathrm{C}$ $k d e$ distribution showed the highest peak at the deeper and wrong depth, while the $6 \mathrm{C} k d e$ distribution resulted in the correct depth. Interestingly, Donner et al. (2013) and Donner et al. (2014) found a similar bimodal sensitivity for centroid depth when inverting real translational measurements for the moment tensor. So, the cause might be in the eventreceiver geometry for the Iranian setting. Based on the two rings of virtual receivers around the source the inversion resulted in unimodal $k d e$ distributions. However, again from $3 \mathrm{C}$ waveform data the possibility to end up with a wrong centroid depth is high. Including rotational data increase the chances to determine a reliable centroid depth. Here, the increase in IG is $175 \%$.

From the summarised results of the generalised inversion in Fig. 6a the panel for the centroid depth is of particular interest. It shows the well-known problem of resolving this parameter from the inversion of $3 \mathrm{C}$ waveform data alone. No matter how well the receivers are distributed around the source, the results always have a low IG. When adding rotations to the inversion, the $k d e$ distribution stretches over a wide range of IG. That means, the centroid depth is almost always more reliably resolved. Depending on the receiver distribution the gain in information can reach a multiple of the one from $3 \mathrm{C}$ data. The reader should note that these are results from a synthetic strike-slip source which includes mainly horizontal slip. For an event including vertical slip the benefit will probably be even better because of the information on the vertical displacement gradient adding valuable constraint on the inversion. 


\subsection{Resolvability of the tectonic mechanism and DC/non-DC parts}

As explained in Sec. 2.1.3 the full seismic moment tensor contains information on several source components, only part of them having a tectonic origin. Depending on the goal of a scientific study, either the DC (tectonic) or the non-DC (ISO and CLVD) part of the moment tensor is the focus. From inverting recordings of translational ground motion alone it is sometimes not possible to resolve the full moment tensor. Scientists constrain the inversion, for example by setting the ISO part to zero or even invert only for the DC part. Other constraints are also possible. When the reliability of the full moment tensor solution can benefit from recordings of rotational ground motions then the different source components should as well.

Based on their 1000 inversions for the generalisation of their study based on the Iranian setting, Donner et al. (2016) have calculated the decomposition of these 1e9 moment tensor solutions (1e6 start solutions for each inversion) and determined DC, ISO, and CLVD percentages as well as strike, dip, and rake angels of the DC part. The overall distribution for the $3 \mathrm{C}$ and the $6 \mathrm{C}$ case were similar. In both cases the $k d e$ peak values for each parameter did not match the value of the target model. Though, for the $6 \mathrm{C}$ case, they found sub-peaks of the $k d e$ distribution at the target values for DC and ISO part as well as for the dip and rake angles.

The entire decomposition result depends on the reliable estimation of the ISO part (diagonal elements of the moment tensor) because this is the very first step in the chosen decomposition scheme (see Sec. 2.1.3). For the scenarios tested (based on a real station distribution and on a virtual grid of receivers), two of the three diagonal moment tensor components showed flaws in their reliability in the $3 \mathrm{C}$ and $6 \mathrm{C}$ case and, hence, the ISO part is flawed as well. The peak values of the $k d e$ distribution for the $3 \mathrm{C}$ and the $6 \mathrm{C}$ case are off by about $35-45 \%$. As a result, all other decomposition parameters do not fit the target value neither. Nevertheless, the reliability of estimating the ISO part still could be improved. Comparing the IG values for the diagonal moment tensor elements from 3C versus $6 \mathrm{C}$ there is an increase by $35 \%$ for the virtual grid of receivers and by $130 \%$ for the real station distribution scenario. In summary that means, adding recordings of rotation into the inversion indeed brings a benefit for the decomposition of the full moment tensor.

This conclusion is particularly significant for non-tectonic events, such as explosions, mining collapse, and volcanic or geothermally induced events. Sometimes this question is connected with a certain political sensitivity, for example in the frame of the Comprehensive NuclearTest-Ban Treaty (CTBT). Several studies have already addressed this question from translational ground motion alone (e.g. Dreger and Woods, 2002; Barth, 2014; Vavrycǔk and Kim, 2014; Gaebler et al., 2019; Mustać et al., 2020, and many more). For the DPRK nuclear test of 2016 Cesca et al. (2017) showed a trade-off between the ISO, vertical CLVD, and DC part. 
Within the ISO part, it is a trade-off between the horizontal $\left(\mathrm{M}_{x x}\right.$ and $\left.\mathrm{M}_{y y}\right)$ and vertical $\left(\mathrm{M}_{z z}\right)$ diagonal moment tensor elements (see also Julian et al., 1998). For tectonic events, several authors could show that the low resolution of the ISO part is inherently related to the often low resolution of the centroid depth (e.g. Sipkin, 1986; Šilený et al., 1992; Kř́žová et al. 2013). Rotational ground motion measurements can address all of these aspects.

\subsection{Influence of noise}

Studies on synthetic data always lack real world complexities in many ways. One of it is the influence of noise contained in real observations. It can arise from inaccuracies in the measurement, for example noise in the seismogram, or from inaccuracies in the theory, for example hypocenter uncertainties, structural model uncertainties. The latter one often can be taken into account by appropriate inversion strategies. For example, by adjusting the inverted frequency range to match uncertainties in the structural model or by grid-searching a specific range of likely values for parameters such as coordinates and centroid depth.

Noise due to measurement inaccuracies is more difficult to take into account due to its correlated nature (Yagi and Fukahata, 2008). Mustać and Tkalčić (2017) showed that erroneous inversion solutions can be the result of unaccounted noise during inversion which leads to overfitting waveform data. In their 2020 study they demonstrated that the non-ISO part of the moment tensor is affected most from this effect. It has direct consequences for the analysis of non-tectonic sources such as explosions.

In the synthetic studies including rotational ground motions summarised here, the synthetic waveform data were biased with Gaussian noise of $10 \%$ of the maximum amplitude observed in the synthetic waveforms. Because the maximum amplitude between translations and rotations differ by several orders of magnitude they were evaluated separately. To investigate the effect of different noise levels during inversion including rotations, Bernauer et al. (2014) have repeated their Bayesian inversion for kinematic source parameters with varying noise levels. Because the study approach provides a relative comparison on $3 \mathrm{C}$ versus $6 \mathrm{C}$ inversion results, it is controlled only by the ratio of the noise level between rotational and translational synthetics. Therefore, they kept the noise level for the translational data constant while increasing the noise level for the rotational data up to five times the level of the translational noise. They conclude that the signal-to-noise ratio just need to be similar in both types of waveform data for the inversion result to benefit from including rotational ground motion measurements. 


\section{Summary}

Most difficulties with the resolution of the seismic moment tensor components during waveform inversion are connected with depth: specific moment tensor components are badly resolved for shallow sources, centroid depth itself, several trade-offs somehow related to depth or depth-dependent components, and sensitivity to the structural model with depth. This is exactly what rotational ground motion can provide: information on the spatial wavefield gradient of the vertical displacement (see Eqs. 16 and 17). As a consequence, when including rotational ground motion into inversion, the resolution (here, meaning accuracy and precision) of the seismic moment tensor components are significantly increased, in some cases by more than $100 \%$. As to be expected, especially the depth-dependent components and the centroid depth could benefit the most. From finite source studies, we also know that the benefit is even increased when we analyse earthquake sources which include vertical rupture instead of a pure strike-slip source mainly rupturing horizontally.

The benefits for the moment tensor resolution was shown on both, synthetic studies and real ADR data. The benefits for the centroid depth and for sources including vertical rupture was shown with synthetic studies only. So far missing but in progress is a study validating the findings on real, direct measurements.

If the single moment tensor components are more reliably determined, then also the decomposition into different source parts is more reliable. Especially the ISO part (volume changes within the source) is fundamental for the entire decomposition process but also the hardest part to resolve from translational waveform data alone.

In summary, the benefits for the moment tensor resolution mainly are due to two reasons:

- Rotational ground motion recordings contain information on the vertical displacement gradient. This information is not available from classical translational surface station recordings. That is an added information to the waveform inversion (beyond simply adding more data).

- Having available six components of ground motion per observation point instead of only three, the amount of amplitude ratios between the components increases by a factor of five (from 3 to 15, enumerative combinatorics). These ratios uniquely reflect the radiation pattern of the source which is hence better constrained.

Or to put it differently: With respect to the radiation pattern, one $6 \mathrm{C}$ observation is equivalent to two $3 \mathrm{C}$ observations at $90^{\circ}$ azimuth from one another.

From inversion theory we know that the inversion result is generally improved when more data of the same type is added to the inversion, even for generally overdetermined inversion problems (Tarantola, 2005). In the few studies performed so far this effect was considered. 
Having six components per observation only half the number of receivers were used for waveform inversion. Still, the same if not higher solution quality was obtained. This result has important implications for hardware installation and maintenance efforts. At some regions of Earth it is simply not possible to establish a dense station network, even more in planetary seismology. Here, recording the entire wavefield in six components can be highly beneficial, not only for source inversion.

Finally, I want to comment on the question whether ADR and direct measurements of rotation are redundant. No, they are not. ADR is valid only in a specific, rather longperiod range of frequencies, depending on the spatial extent of the receiver array used to calculate them. In contrast, direct measurements cover a very broad range of frequencies, meanwhile even with portable devices. Also, ADR is a spatial averaged measure and it is not entirely clear if it contains the same physical information than a direct point measurement, for example local side effects are averaged out during the calculation. Due to the same reason ADR measurements can not be used for tilt corrections.

\section{Outlook}

The findings from the currently few studies are very promising concerning the improved reliability of the resulting moment tensor from inverting rotational and translational waveforms together. They suggest that some well- and long-known difficulties in regional waveform inversion for seismic moment tensors can be tackled quite well with the relatively new measurement of rotational ground motion. However, some aspects have not yet been investigated. The most important aspect is the question whether the results can be replicated from real, directly measured data. The study using ADR data is a pointer in this direction. However, as already explained ADR data is not entirely comparable with direct measurements. One specific aspect of ADR data is their spatial averaging over the array area. Thus, they average out local side effects. In contrast, meanwhile we know that rotational ground motion are much more sensitive to small-scale structural heterogeneities in the vicinity of the receiver. While we often can ignore or compensate local side effects when analysing translational ground motion, we probably need to correct rotational ground motion for these effects before we can analyse them. The introduction of the strain-rotation coupling vector $\boldsymbol{J}$ is a good approach to do so. However, it was not applied yet on real data as preparation for further studies such as waveform inversion for moment tensors.

Another aspect of ADR data is their reliable range of frequencies, depending on the spatial extend of the translational receiver array used to calculate them (see Sec. 2.2.2). Real arrays, i. e. particularly designed for array processing purposes, usually have quite small interreceiver distances of a few kilometres, resulting in relatively high reliable frequency ranges, 
roughly higher than $0.005 \mathrm{~Hz}$. These frequencies are still quite small for regional waveform inversion. Using 'normal' receiver networks to calculate ADR the network extend quickly becomes large and the resulting reliable frequencies very small. This is in contradiction to the frequencies usually used in regional waveform inversion for moment tensors $(f \leq$ $0.01 \mathrm{~Hz}$ ). Nevertheless, it is not investigated yet whether both types of measurement could complement each other in waveform inversion for moment tensors. If done with proper care for the limitations of the method, ADR data might be a useful addendum to the waveform inversion for moment tensors.

Most of the findings summarised in this article are from studies based on synthetic data. They are lacking real world complexities in many ways, mainly due to missing correlated noise (see Secs. 3.3 and 3.6). Therefore, the most important next step in investigating the benefits of rotational ground motion for waveform inversion for moment tensors is the validation from real, direct measurements. Effects of uncertainties in structural model, local side effects and strain rotation coupling, uncertainties in epicenter versus hypocenter location, measurement errors, and used frequency range can be investigated thoroughly only on real data.

In addition, the studies so far investigated the benefits of rotational ground motion either in a setting with an entire network of $6 \mathrm{C}$ receivers or a sparse network of only two or three $6 \mathrm{C}$ receivers. Not answered yet is the question how much benefit it will bring to expand an existing network of translational receivers with only one or two rotational receivers. This question is interesting not only for the regional but also for the local distance range. Given the promising findings so far, it might be possible to circumvent limitations due to relations between frequency, structure, and magnitude (see Sec. 3.3) and expand the waveform inversion to much lower magnitude thresholds. Such expanded catalogues of earthquake mechanisms broadens the underlying data base for subsequent seismotectonic studies enormously. Particularly in regions with low seismicity rate, where the number of medium to large sized earthquakes over time is low, this is a possibility to gain further insights into tectonic processes.

Further investigation is also needed on the topic of moment tensor decomposition, especially on the reliable determination of the difficult to obtain ISO part. Thus, we can learn more about the physical processes within the source beyond the pure tectonic shear rupture; that is in volcanic areas and areas with swarm activity as well as induced source processes connected to geothermal facilities, mining activities, and oil/gas production. Increasing the reliability of the decomposition by incorporating rotational ground motion contributes strongly to distinguishing between tectonic, explosive and induced sources and analysing them.

In Sec. 2.1.1 I show that the equation for the general elastodynamic source (Eq. 1) is simplified when external forces such as gravitation are neglected. That is net forces and torques are vanishing and thus the moment tensor becomes symmetric. Several authors showed that these are overly restrictive assumptions and real sources indeed include net 
forces and torque components. Abreu et al. (2018) have developed a framework for a more realistic, asymmetric moment tensor using micropolar theory. It allows an independent rotation within the source (spin) which is different form the continuum, rigid body rotation. The theory enables to better account for deformation by including material rotations during the rupture and thus better constrains physical processes within the source. The theory still needs to be validated against real measurements.

\section{Acknowledgement}

I thank the editor C. Schmelzbach for the opportunity to write this review and for his support throughout the writing and publishing process. Reviewer G. Hillers' constructive comments considerably helped to improve the accessibility of the manuscript.

\section{References}

R. Abreu, S. Durand, and C. Thomas. The asymmetric seismic moment mensor in micropolar media. Bull. Seismol. Soc. Am., 108(3A):1160-1170, 2018.

P. Adamova and J. Šilený. Non-double-couple earthquake mechanism as an artefact of the point-source approach applied to a finite-extent focus. Bull. Seismol. Soc. Am., 100:447457, 2010.

K. Aki and P. G. Richards. Quantitative seismology. University Science Book, second edition, 2002.

N. Aso, K. Ohta, and S. Ide. Mathematical review on source-type diagrams. Earth, Planets, and Space, 68:52, 2016.

G. Backus and M. Mulcahy. Moment tensors and other phenomenological descriptions of seismic sources - I. Continuous displacements. Geophys. J. R. astr. Soc., 46:341-361, 1976.

G. Backus. Interpreting the seismic glut moments of total degree two or less. Geophys. J.

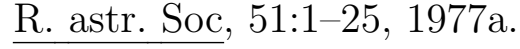

G. Backus. Seismic sources with observable glut moments of spatial degree two. Geophys. J. R. astr. Soc., 51:27-45, 1977b.

A. Barth. Significant release of shear energy of the North Korean nuclear test on February 12, 2013. J. Seismol., 18(3):605-615, 2013. 
M. Bernauer, A. Fichtner, and H. Igel. Measurements of translation, rotation and strain: new approaches to seismic processing and inversion. J. Seismol., 16:669-681, 2012.

M. Bernauer, A. Fichtner, and H. Igel. Reducing nonuniqueness in finite source inversion using rotational ground motions. J. Geophys. Res., 119:4860-4875, 2014.

F. Bernauer, J. Wassermann, F. Guattari, A. Frenois, A. Bigueur, A. Gaillot, E. de Toldi, D. Ponceau, K. U. Schreiber, and H. Igel. BlueSeis3A - full characterization of a 3C broadband rotational seismometer. Seismol. Res. Lett., 89(2A):620-629, doi: 10.1785/0220170143.

F. Bernauer, K. Behnen, J. Wassermann, S. Egdorf, H. Igel, S. Donner, K. Stammler, M. Hoffmann, P. Edme, D. Sollberger, C. Schmelzbach, J. Robertsson, P. Paitz, J. Igel, K. Smolinski, A. Fichtner, Y. Rossi, G. Izgi, D. Vollmer, E. P. S. Eibl, S. Buske, C. Veress, F. Guattari, T. Laudat, L. Mattio, O. Sébe, S. Olivier, C. Lallemand, B. Brunner, A. T. Kurzych, M. Dudek, L. R. Jaroszewicz, J. K. Kowalski, P. A. Bońkowski, P. Bobra, Z. Zembaty, J. Vackář, J. Málek, and J. Brokesova. Rotation, strain, and translation sensors performance tests with active seismic sources. Sensors, 21(1):264, 2021, https://doi.org/10.3390/s21010264.

M. Beyreuther, R. Barsch, L. Krischer, T. Megies, Y. Behr, and J. Wassermann. ObsPy: A Python toolbox for seismology Seismol. Res. Lett., 81:530-533, 2010.

P. Bodin, J. Gomberg, S. K. Singh, and M. Santoyo Dynamic deformations of shallow sediments in the Valley of Mexico, Part I: three-dimensional strains and rotations recorded on a seismic array. Bull. Seismol. Soc. Am., 87:528-539, 1997.

J. Braunmiller, U. Kradolfer, M. Baer, and D. Giardini. Regional moment tensor determination in the European-Mediterranean area - initial results. Tectonophysics, 356:5-22, 2002.

J. Brokešová, J. Málek, and J. r. Evans. Note: Rotaphone, a new self-calibrated six-degreeof-freedom seismic sensor. Rev. Sci. Instrum., 83(8):086108-1-086108-3, 2012.

J. Brokešová and J. Málek. Rotaphone, a self-calibrated six-degree-of-freedom seismic sensor and its strong-motion records. Seismol. Res. Lett., 84(5):737-744, 2013.

B. Bukchin. Specific features of surface wave radiation by shallow sources. Phys. Solid Earth, 42:712-717, 2006.

B. Bukchin, E. Clévédé, and A. Mostinskiy. Uncertainty of moment tensor determination from surface wave analysis for shallow earthquakes. J. Seismol, 14:601-614, 2010. 
Y. Capdeville, P. Cupillard, and S. Singh. An introduction to the two-scale homogenization method for seismology. Advances in Geophysics, 61:217-306, 2020.

S. Cesca and S. Heimann. A practical on moment tensor inversion using the Kiwi tools. In: P. Bormann, editor, New Manual of Seismological Observatory Practice 2 (NMSOP-2), EX 3.6. Potsdam: Deutsches GeoForschungsZentrum GFZ, 2013.

S. Cesca, A. Rohr and T. Dahm. Discrimination of induced seismicity by full moment tensor inversion and decomposition. J. Seismol., 17:147-163, 2013.

S. Cesca, S. Heimann, M. Kriegerowski, J. Saul, and T. Dahm. Moment tensor inversion of nuclear explosions: what can we learn from the 6 January and 9 September 2016 nuclear tests, North Korea? Seismol. Res. Lett., 88(2A):300-310, 2017.

C. Cesca and S. Heimann. Challenges in regional moment tensor resolution and interpretation. In: S. D'Amico, editor, Moment tensor solutions - A useful tool for seismotectonics, pages 163-181. Springer International Publishing, 2018.

A. Cochard, H. Igel, B. Schuberth, W. Suryanto, A. Velikoseltsev, U. Schreiber, J. Wassermann, F. Scherbaum, D. Vollmer. Rotational motions in seismology: theory, observation, simulation. In: R. Teisseyre, E. Majewski, M. Takeo, editors, Earthquake source asymmetry, structural media and rotation effects, pages 391-411. Springer Verlag, Berlin/Heidelberg, 2006.

B. M. Covellone and B. Savage. A quantitative comparison between 1D and 3D source inversion methodologies: Application to the Middle East. Bull. Seism. Soc. Am., 102(5):2189 2199, 2012.

R. Cowsik, T. Madziwa-Nussinov, K. Wagoner, D. Wiens, and M. Wysession. Performance characteristics of a rotational seismometer for near-field and engineering applications. Bull. Seismol. Soc. Am., 99(2B):1181-1189, 2009.

T. Dahm. Relativmethoden zur Bestimmung der Abstrahlcharakteristik von seismischen Quellen. PhD Thesis, University of Karlsruhe, Germany, 1993.

T. Dahm, G. Manthei, and J. Eisenblätter. Automated moment tensor inversion to estimate source mechanisms of hydraulically induced micro-seismicity in salt-rock. Tectonophysics, 306:1-17, 1999.

T. Dahm, F. Krüger, K. Stammler, K. Klinge, R. Kind, K. Wylegalla and J.-R. Grasso. The 2004 Mw 4.4 Rotenburg, northern Germany, earthquake and its possible relationship with gas recovery. Bull. seism. Soc. Am., 97(3):691-704, 2007. 
F. Krüger and T. Dahm. Moment tensor inversion and moment tensor interpretation. In: P. Bormann, editor, New Manual of Seismological Observatory Practice 2 (NMSOP-2), IS 3.9. Potsdam: Deutsches GeoForschungsZentrum GFZ, 2014.

T. Dahm, S. Heimann, S. Funke, S. Wendt, I. Rappsilber, D. Bindi, T. Plenefisch, and F. Cotton. Seismicity in the block mountains between Halle and Leipzig, central Germany: centroid moment tensors, ground motion simulation, and felt intensities of two $\mathrm{M} \sim 3$ earthquakes in 2015 and 2017. J. Seismol., 22:985-1003, 2018.

S. D'Amico. Moment tensor solutions - A useful tool for seismotectonics. Springer International Publishing, 2018.

B. Delouis and D. Legrand. Focal mechanism determination and identification of the fault plane of earthquakes using only one or two near-source seismic recordings. Bull. Seismol. Soc. Am., 89:1558-1574, 1999

S. Donner, D. Rößler, F. Krüger, A. Ghods, and M. R. Strecker. Segmented seismicity of the Mw 6.2 Baladeh earthquake sequence (Alborz mountains, Iran) revealed from regional moment tensors. J. Seismol., 17:925-959, 2013.

S. Donner, F. Krüger, D. Rößler, and A. Ghods. Combined inversion of broad-band and short-period waveform data for regional moment tensors: A case study in the Alborz mountains, Iran. Bull. Seismol. Soc. Am., 104:1358-1373, 2014.

S. Donner, M. Bernauer, and H. Igel. Inversion for seismic moment tensors combining translational and rotational ground motions. Geophys. J. Int., 207:562-570, 2016.

S. Donner, C.-J. Lin, C. Hadziioannou, A. Gebauer, F. Vernant, D. C. Agnew, H. Igel, K. U. Schreiber, and J. Wassermann. Comparing direct observation of strain, rotation, and displacement with array estimates at Piñon Flat Observatory, California. Seismol. Res. Lett., 88(4):1107-1116, 2017.

S. Donner, H. Igel, C. Hadziioannou, and the Romy group. Retrieval of the seismic moment tensor from joint measurements of translational and rotational ground motions: Sparse networks and single stations. In: S. D'Amico, editor, Moment tensor solutions: A useful tool for seismotectonics, Springer International Publishing, pp. 263-280, 2018.

S. Donner, M. Mustać, B. Hejrani, H. Tkalčić, and H. Igel. Seismic moment tensors from synthetic rotational and translational ground motion: Green's functions in 1-D versus 3-D. Geophys. J. Int., 223:161-179, 2020. 
D. Doornbos. Seismic moment tensors and kinematic source parameters. Geophys. J. R. astr. Soc., 69:235-251, 1982.

D. Dreger and D. Helmberger. Source parameters of the Sierra Madre earthquake from regional and local body waves. Geophys. Res. Lett., 18(11):2015-2018, 1991.

D. Dreger and D. Helmberger. Determination of source parameters at regional distances with three-component sparse network data. J. Geophys. Res., 98:8107-8125, 1993.

D. Dreger and B. Woods. Regional distance seismic moment tensors of nuclear explosions. Tectonophysics, 356:139-156, 2002.

H. Dufumier and M. Cara. On the limits of linear moment tensor inversion of surface wave spectra. Pure appl. Geophys., 145:235-257, 1995.

Z. Duputel, L. Rivera, Y. Fukahata, and H. Kanamori. Uncertainty estimations for seismic source inversion. Geophys. J. Int., 190:1243-1256, 2012.

A. M. Dziewonski and F. Gilbert. Temporal variation of the seismic moment tensor and the evidence of precursive compression for two deep earthquakes. Nature, 247:185-188, 1974.

A. M. Dziewonski, T.-A. Chou, and J. H. Woodhouse. Determination of earthquake source parameters from waveform data for studies of global and regional seismicity. J. Geophys. Res., 86(B4):2825-2852, 1981.

D. Eberhart-Phillips. Three-dimensional P and S velocity structure in the Coalinga region, California. J. Geophys. Res., 95:15,343-15,363, 1990.

G. Ekström, A. M. Dziewonski, and J. M. Steim. Single station CMT: application to the Michoacan, Mexico, earthquake of September 19, 1985. Geophys. Res. Let., 13:173-176, 1986 .

G. Ekström, M. Nettles, and A. M. Dziewonski. The global CMT project 2004-2010: Centroid-moment tensors for 13,017 earthquakes. PEPI, 200-201:1-9, 2012.

G. Fan and T. Wallace. The determination of source parameters for small earthquakes from a single, very broadband seismic station. Geophys. Res. Let., 18:1385-1388, 1991.

A. Fichtner and H. Igel. Sensitivity densities for rotational ground-motion measurements. Bull. Seismol. Soc. Am., 99(2B):1302-1314, 2009.

A. Fichtner and H. Tkalčić. Insights into the kinematics of a volcanic caldera drop: Probabilistic finite-source inversion of the 1996 Bardarbunga, Iceland, earthquake. Earth Planet. Sci. Lett. 297(3-4):607-615, 2010. 
T. J. Fitch, D. W. McCowan, and M. W. Shields. Estimation of the seismic moment tensor from teleseismic body wave data with application to intraplate and mantle earthquakes. J. Geophys. Res., 85:3817-3828, 1980.

S. R. Ford, D. S. Dreger, and W. R. Walter. Network sensitivity solutions for regional moment tensor inversions. Bull. Seismol. Soc. Am., 100(5A):1962-1970, 2010.

C. Frohlich. Note concerning non-double-couple source components from slip along surfaces of revolution. J. Geophys. Res., 95(B5):6861-6866, 1990.

C. Frohlich. Earthquakes with non-double-couple mechanisms. Science, 264:804-809, 1994.

P. Gaebler, L. Ceranna, N. Nooshiri, A. Barth, S. Cesca, M. Frei, I. Grünberg, G. Hartmann, K. Koch, C. Pilger, J. O. Ross, and T. Dahm. A multi-technology analysis of the 2017 North Korean nuclear test. Solid Earth, 10(1):59-78, 2019.

J. W. Gephart and D. W. Forsyth An improved method for determining the regional stress tensor using earthquake focal mechanism data: Application to the San Francisco Earthquake Sequence. J. Geophys. Res., 89(B11):9305-9320, 1984

F. Gilbert. Excitation of the normal modes of the Earth by earthquake sources. Geophys. J. R. astr. Soc., 22:223-226, 1970.

F. Gilbert. Derivation of source parameters from low-frequency spectra. Phil. Trans. R. SOC., 274:369-371, 1973.

F. Gilbert and A. M. Dziewonski. An application of normal mode theory to the retrieval of structural parameters and source mechanisms from seismic spectra. Philosophical Transactions of the Royal Society of London; Series A, Mathematical and Physical Sciences, 278(1280):187-269, 1975.

R. W. Graves and D. J. Wald. Resolution analysis of finite fault source inversion using one- and three-dimensional Green's functions 1. Strong motions. J. Geophys. Res., 106(B5):8745-8766, 2010.

M. T. Gudmundsson, K. Jónsdóttir, A. Hooper, E. P. Holohan, S. A. Halldórsson, B. G. Ófeigsson, S. Cesca, K. S. Vogfjörd, F. Sigmundsson, T. Högnadóttir, P. Einarsson, O. Sigmarsson, A. H. Jarosch, K. Jónasson, E. Magnússon, S. Hreinsdóttir, M. Bagnardi, M. M. Parks, V. Hjörleifsdóttir, F. Pálsson, T. R. Walter, M. P. J. Schöpfer, S. Heimann, H. I. Reynolds, S. Dumont, E. Bali, G. H. Gudfinnsson, T. Dahm, M. J. Roberts, M. Hensch, J. M. C. Belart, K. Spaans, S. Jakobsson, G. B. Gudmundsson, H. M. Fridriksdóttir, V. Drouin, T. Dürig, G. Aðalgeirsdóttir, M. S. Riishuus, G. B. M. Pedersen, T. van Boeckel, 
B. Oddsson, M. A. Pfeffer, S. Barsotti, B. Bergsson, A. Donovan, M. R. Burton, and A. Aiuppa. Gradual caldera collapse at Bárdarbunga volcano, Iceland, regulated by lateral magma outflow. Science, 353(6296), aaf9899-1-aaf8988-8, 2016.

C. Haberland, A. Rietbrock, D. Lange, K. Bataille, and T. Dahm. Structure of the seismogenic zone of the southcentral Chilean margin revealed by local earthquake traveltime tomography. J. Geophys. Res., 114:B01317, 2009.

J. C. Harrison. Cavity and topographic effects in tilt and strain measurement. J. geophys. Res., 81(2):319-328, 1976.

N. A. Haskell. Radiation pattern of surface waves from point sources in a multi-layered medium. Bull. Seism. soc. Am., 54(1):377-393, 1964.

W. K. Hastings. Monte Carlo sampling methods using Markov chains and their applications. Biometrika, 57:97-109, 1970.

O. Heidbach, M. Rajabi, X. Cui, K. Fuchs, B. Müller, J. Reinecker, K. Reiter, M. Tingay, F. Wenzel, F. Xie, M. Ziegler, M.-L. Zoback, and M. Zoback. The World Stress Map database release 2016: Crustal stress pattern across scales. Tectonophysics, 744:484-498, 2018.

B. Hejrani, H. Tkalčić and A. Fichtner. Centroid moment tensor catalogue using a 3-D continental scale Earth model: Application to earthquakes in Papua New Guinea and the Solomon Islands. J. Geophys. Res., 122:5517-5543, doi: 10.1002/2017JB014230, 2017.

B. Hejrani and H. Tkalcic. Resolvability of the centroid-moment-tensors for shallow seismic sources and improvements from modelling high-frequency waveforms. J. Geophys. Res., 125(7):e2020JB019643, 2020.

R. Herrmann, H. Benz, and C. Ammon. Monitoring the earthquake source process in North America. Bull. Seismol. Soc. Am., 101:2609-2625, 2011.

M. Hingee, H. Tkalčić, A. Fichtner and M. Sambridge. Seismic moment tensor inversion using a 3-D structural model: applications for the Australian region. Geophys. J. Int., 184:949-964, doi: 10.1111/j.1365-246X.2010.04897.x, 2011.

V. Hjörleifsdóttir and G. Ekström. Effects of three-dimensional Earth structure on CMT earthquake parameters. PEPI, 179:178-190, 2010.

H. Huang, V. Agafonov, and H. Yu. Molecular electric transducers as motion sensors: A review Sensors, 13(4):4581-4597, 2013. 
B. S. Huang. Ground rotational motions of the 1991 Chi-Chi, Taiwan, earthquake as inferred from dense array observations. Geophys. Res. Lett., 30(6):1307-1310.

J. A. Hudson, R. G. Pearce, and R. M. Rogers. Source type plot for inversion of the moment tensor. J. Geophys. Res., 94(B1):765-774, 1989.

G. A. Ichinose, S. R. Ford, and R. J. Mellors. Regional moment tensor inversion using rotational observations. J. Geophys. Res., 126:e2020JB020827, 2021.

H. Igel, K. U. Schreiber, B. Schuberth, A. Flaws, A. Velikoseltsev, and A. Cochard. Observation and modelling of rotational motions induced by distant large earthquakes: the $\mathrm{M}$ 8.1 Tokachi-oki earthquake September 25, 2003. Geophys. Res. Lett. 32:L08309, 2005.

H. Igel, M. Bernauer, J. Wassermann, and K.U. Schreiber. Rotational Seismology: Theory, Instrumentation, Observations, Applications. In: R.A. Meyers, editor Encyclopedia of Complexity and Systems Science, pages 391-411. Springer, Berlin, Heidelberg, 2015.

D. Inazu, N. Pulido, E. Fukuyama, T. Saito, J. Senda, and H. Kumagai. Near-field tsunami forecast system based on near real-time seismic moment tensor estimation in the regions of Indonesia, the Philippines, and Chile. Earth, Planets and Space, 68:73, 2016.

P. Jedlička, J. T. Kozák, J. R. Evans, and C. R. Hutt. Designs and test results for three new rotational sensors. J. Seismol., 16(4):639-647, 2012.

L. R. Jaroszewicz, Z. Krajewski, L. Solarz, and R. Teisseyer Application of the fibre-optic Sagnac interferometer in the investigation of seismic rotational waves. Institute of Physics Publishing, 17:1186-1193.

M. L. Jost and R. B. Herrmann. A student's guide to and review of moment tensors. Seismol. Res. Let., 60:37-57, 1989.

B. R. Julian, A. D. Miller, and G. R. Foulger. Non-double-couple earthquakes - 1. Theory. Reviews of Geophysics, 36(4):525-549, 1998.

H. Kanamori and J. W. Given. Use of long-period surface waves for rapid determination of earthquake source parameters - 2. Preliminary determination of source mechanisms of large earthquakes $\left(\mathrm{M}_{S} \leq 6.5\right)$ in 1980. PEPI, 30:260-268, 1982.

H. Kanamori and L. Rivera. Source inversion of W phase: speeding up the seismic tsunami warning. Geophys. J. Int., 175:222-238, 2008.

Y. Kim, Q. Liu and J. Tromp. Adjoint centroid-moment tensor inversions. Geophys. J. Int. 186(1):264-278, 2011. 
G. King and R. Bilham. Tidal tilt measurement in Europe. Nature, 243(5402):74-75. 1973.

J. Kinscher, F. Krüger, H. Woith, B. G. Lühr, E. Hintersberger, T. S. Irmak, and S. Bariş. Seismotectonics of the Armutlu peninsula (Marmara Sea, NW Turkey) from geological field observation and regional moment tensor inversion. Tectonophysics, 608:980-995, 2013.

L. Knopoff and M. J. Randall. The compensated linear-vector dipole: a possible mechanism for deep earthquakes. J. Geophys. Res., 75:4957-4963, 1970.

M. L. Kohl and J. Levine. Measurement and interpretation of tidal tilts in a small array. J. geophys. Res., 100(B3):3929-3941,1995.

L. Krischer, T. Megies, R. Barsch, M. Beyreuther, T. Lecocq, C. Caudron, and J. Wassermann. ObsPy: A bridge for seismology into the scientific Python ecosystem. Comput. Sci. Disc., 8:014003, 2015.

D. Křížová, J. Zahradníck, and A. Kiratzi. Resolvability of isotropic component in regional seismic moment tensor inversion. Bull. seism. Soc. Am., 103:2460-2473, 2013.

F. Krüger and G. Bock. Moment-tensor determination and decomposition. In: P. Bormann, editor, New Manual of Seismological Observatory Practice 2 (NMSOP), EX 3.5. Potsdam: Deutsches GeoForschungsZentrum GFZ, 2009.

F. Krüger and F. Scherbaum. Short Note: The 29 September 1969, Ceres, South Africa, earthquake: Full waveform moment tensor inversion for point source and kinematic source parameters. Bull. Seism. soc. Am., 140(1):576-581, 2014.

D. Kühn and V. Vavryčuk. Determination of full moment tensors of microseismic events in a very heterogeneous mining environment. Tectonophysics, 589:33-43, 2013.

D. Kühn, S. Heimann, M. P. Isken, E. Ruigrok, and B. Dost. Probabilistic moment tensor inversion for hydrocarbon-induced seismicity in the Groningen gas field, The Netherlands, Part 1: Testing. Bull. Seismol. soc. Am., 110(5):2095-2111, 2020.

C. A. Langston. Spatial gradient analysis for linear seismic arrays. Bull. Seismol. soc. Am., 97:265-280, 2007a.

C. A. Langston. Wave gradiometry in two dimensions. Bull. Seismol. soc. Am., 97:401-416, 2007b.

C. A. Langston. Wave gradiometry in the time domain. Bull. Seismol. soc. Am., 97:926-933, 2007c. 
C. A. Langston. Calibrating dense spatial arrays for amplitude statics and orientation errors. J. Geophys. Res., 123:3849-3870, 2018.

W.H.K. Lee, B.-S. Huang, C. A. Langston, C.-J. Lin, C.-C. Liu, T.-C. Shin, T.-L. Teng, and C.-F. Wu. Review: progress in rotational ground motion observations from explosions and local earthquakes in Taiwan. Bull. Seismol. soc. Am., 99(2b):958-967, 2009.

W.H.K. Lee, J.R. Evans, B.-S. Huang, S.R. Hutt, C.-J. Lin, C.-C. Liu, and R.L. Nigbor. Measuring rotational ground motions in seismological practice. In: P. Bormann, editor, New Manual of Seismological Observatory Practice 2 (NMSOP-2), IS 5.3. Potsdam: Deutsches GeoForschungsZentrum GFZ, 2011.

H. C. Lefèvre. The fibre-optic gyroscope. Artech House, London, United Kingdom, 2014.

D. Legrand, S. Kaneshima and H. Kawakatsu. Moment tensor analysis of near-field broadband waveforms observed at Aso Volcano, Japan. J. Volc. Geotherm. Res., 101(1-2):155$169,2000$.

Z. Li and M. van der Baan. Tutorial on rotational seismology and its applications in exploration geophysics. Geophysics, 82(5):W17-W30, 2017.

C. Liang and C. A. Langston Wave gradiometry for USArray: Rayleigh waves J. Geophys. Res., 114:B02308, 2009.

C.-J. Lin, W.-G. Huang, H.-P. Huang, B.-S. Huang, C.-S. Ku, and C.-C. Liu. Investigation of array derived rotation in TAIPEI 101. J. Seismol., 16:1-11, 2012.

T. Megies, M. Beyreuther, R. Barsch, L. Krischer, and J. Wassermann. ObsPy - What can it do for data centers and observatories. Ann. Geophys., 54:47-58, 2011.

N. Metropolis and S. Ulam. The monte carlo method. Journal of the American Statistical Association, 44:335-341, 1949.

N. Metropolis, M. N. Rosenbluth, A. H. Teller, and E. Teller. Equation of state calculations by fast computing machines. Journal of Chemical Physics, 21:1087-1092, 1953.

D. P. McLeod, G. E. Stedman, T. H. Webb, and K. U. Schreiber. Comparison of standard and ring laser rotational seismograms. Bull. Seismol. Soc. Am., 88:1495-1503,1998.

S. Mousavi, K. Bauer, M. Korn, and B. Heijrani. Seismic tomography reveals a mid-crustal intrusive body, fluid pathways and their relation to the earthquake swarms in West Bohemia/Vogtland. Geophys. J. Int., 203:1113-1127, 2015. 
M. Mustać and H. Tkalčić. On the use of data noise as a site-specific weight parameter in a hierarchical Bayesian moment tensor inversion: the case study of the Geysers and Long Valley Caldera earthquakes. Bull. seism. Soc. Am, 107:1914-1922, 2017.

M. Mustać, B. Hejrani, H. Tkalčić, S. Kim, S.-J. Lee, and C. S. Chow. Large isotropic component in the source mechanism of the 2013 Democratic People's Republic of Korea nuclear test revealed via a hirarchical Bayesian inversion. Bull. seism. Soc. Am., 110(1):166-177, 2020.

M. Nettles and G. Ekström. Faulting mechanism of anomalous earthquakes near Bádarbunga Volcano, Iceland. J. Geophys. Res., 103(B8):17973-17983, 1998.

R. L. Nigbor. Six-degree-of-freedom ground-motion measurement. Bull. Seismol. Soc. Am., 84(5):1665-1669, 1994.

R. L. Nigbor, J. R. Evans, and C. R. Hutt. Laboratory and field testing of commercial rotational seismometers. Bull. Seismol. Soc. Am., 99(2b):1215-1227, 2009.

T. Nissen-Meyer, M. van Driel, S. C. Stähler, K. Hosseini, S. Hempel, L. Auer, A. Colombi, and A. Fournier. AxiSEM: broadband 3-D seismic wavefield in axisymmetric media. Solid Earth, 5:425-445, 2014.

A. Pancha, T. H. Webb, G. E. Stedman, D. P. McLeod, and K. U. Schreiber. Ring laser detection of rotations from teleseismic waves. Geophys. Res. Lett., 27:3553-3556, 2000.

N. D. Pham, H. Igel, J. de la Puente, M. Käser, and M. A. Schoenberg. Rotational motions in homogeneous anisotropic elastic media. Geophysics, 75:D47-D56, 2010.

B. Pierson, D. Laughlin, and B. Brune. Seismic MHD (SMHD) - Rotational sensor development and deployment. 4th IWGoRS Meeting, Tutzing Germany, 20-23 June 2016.

M. Reinwald, M. Bernauer, H. Igel, and S. Donner. Improved finite-source inversion through joint measurements of rotational and translational ground motions: a numerical study. Solid Earth, 7:1467-1477, 2016.

J. O. A. Robertsson and A. Curtis. Wavefield separation using densely deployed three-component single-sensor groups in land surface-seismic recordings. Geophysics, 67(5):1624-1633, 2002.

S. Schippkus, H. Hausmann, Z. Duputel, G. Bokelmann and AlpArray Working Group. The Alland earthquake sequence in Eastern Austria: Shedding light on tectonic stress geometry in a key area of seismic hazard. Austrian Journal of Earth Sciences, 112(2):182-194, 2020. 
W. Schlüter. Schwingungsart und Weg der Erdbebenwellen. Beiträge zur Geophysik, 5:314359, 1903.

C. Schmelzbach, D. Donner, H. Igel, D. Sollberger, T. Taufiqurrahman, F. Bernauer, M. Häusler, C. van Renterghem, J. Wassermann, and J.O.A. Robertsson. Advances in 6C seismology: Applications of combined translational and rotational motion measurements in global and exploration seismology. Geophysics, 83(3):WC53-WC69, doi: 10.1190/GEO2017-0492.1, 2018.

K. U. Schreiber, J. N. Hautmann, A. Velikoseltsev, J. Wassermann, H. Igel, J. Otero, F. Vernon, and J.-P. R. Wells. Ring Laser Measurements of Ground Rotations for Seismology. Bull. Seismol. Soc. Am., 99(2b):1190-1198, 2009.

C. E. Shannon. A mathematical theory of communication. Bell System Tech. J., 27:379-423, 1948.

A. Shuler, G. Ekström, and M. Nettles. Physical mechanisms for vertical-CLVD earthquakes at active volcanoes. J. Geophys. Res., 118:1569-1586, 2013.

A. T. Sen, S. Cesca, M. Bischoff, T. Meier and T. Dahm. Automated full moment tensor inversion of coal mining-induced seismicity. Geophys. J. Int., 195(2):1267-1281, 2013.

P. Shi, D. Angus, A. Nowacki, S. Yuan, and Y. Wang. Microseismic full waveform modeling in anisotropic media with moment tensor implementation. Surveys in Geophysics, 39:567611, 2018.

J. Šilený. Regional moment tensor uncertainty due to mismodeling of the crust. Tectonophysics, 383:133-147, 2004.

P. G. Silver and T. H. Jordan. Optimal estimation of scalar seismic moment. Geophys. J. R. astr. Soc., 70:755-787, 1982.

P. Silver. Retrieval of source-extent parameters and the interpretation of corner frequency. Bull. seism. Soc. Am., 73:1499-1511, 1983.

S. Singh, Y. Capedeville, and H. Igel. Correcting wavefield gradients for the effects of local small-scale heterogeneities. Geophys. J. Int., 220:996-1011, 2020.

J. Šilený, G. F. Panza, and P. Campus. Waveform inversion for point source moment tensor retrieval with variable hypocentral depth and structural model. Geophys. J. Int., 109:259274, 1992. 
S. A. Sipkin. Interpretation of non-double-douple earthquake mechanisms derived from moment tensor inversion. J. Geophys. Res., 91:531-547, 1986.

D. Sollberger, H. Igel, C. Schmelzbach, P. Edme, D.-J. van Manen, F. Bernauer, S. Yuan, J. Wassermann, U. Schreiber und J. O. A. Robertsson. Seismological processing of six degree-of-freedom ground-motion data. Sensors, 20:6904, 2020.

P. Spudich and J. B. Fletcher. Observation and prediction of dynamic ground strains, tilts, and torsions caused by the Mw 6.0 2004 Parkfield, California, earthquake and aftershocks, derived from UPSAR array observations. Bull. Seismol. Soc. Am., 98:1898-1914, 2008.

P. Spudich and J. B. Fletcher. Short Note: Software for Inference of Dynamic Ground Strains and Rotations and Their Errors from Short Baseline Array Observations of Ground Motions. Bull. Seismol. Soc. Am., 99(2b):1480-1482, 2009.

P. Spudich, L. K. Steck, M. Hellweg, J. B. Fletcher, and L. M. Baker. Transient stresses at Parkfield, California, produced by the M 7.4 Landers earthquake of June 28, 1992 observations from the UPSAR dense seismograph array. J. Geophys. Res., 100:675-690, 1995.

B. Stump and L. Johnson. Higher-degree moment tensors - the importance of source finiteness and rupture propagation on seismograms. Geophys. J. R. astr. Soc., 69:721-741, 1982.

W. Suryanto, H. Igel, J. Wassermann, A. Cochard, B. Schuberth, D. Vollmer, F. Scherbaum, U. Schreiber, and A. Velikoseltsev. First comparison of array-derived rotational ground motions with direct ring laser measurements. Bull. Seismol. Soc. Am., 96:2059-2071, 2006.

M. Takeo. Ground rotational motions recorded in near-source region of earthquakes. Geophys. Res. Let., 25(6):789-792, 1998.

W. Tape and C. Tape. A geometric comparison of source-type plots for moment tensors. Geophys. J. Int., 190(1):499-510, 2012.

A. Tarantola. Inverse problem theory and methods for model parameter estimation, 2nd edn. Society for Industrial and Applied Mathematics, Philadelphia, Penn., 2005.

C. H. Thurber. Local earthquake tomography: velocities and Vp/Vs - theory. In: H. M. Iyer and K. Hirahara, editors, Seismic Tomography: Theory and Practice, pp. 563-583, Chapman and Hall, London, 1993.

G. Toyokuni and H. Takenaka. FDM computation of seismic wavefield for an axisymmetric Earth with a moment tensor point source. Earth, Planets and Space, 58:e29-e32, 2006. 
A. Udías. Principles of Seismology. Cambridge University Press, ISBN 978-0-521-62478-7, 1999.

A. Udías, R. Madariaga, and E. Buforn. Source Mechanisms of Earthquakes: Theory and Practice. Cambridge University Press, ISBN 978-1-107-04027-4, 2014.

M. van Driel, J. Wassermann, M. F. Nader, B. S. A. Schuberth, and H. Igel. Strain rotation coupling and its implications on the measurement of rotational ground motions. J. Seismol., 16(4):657-668, 2012.

M. van Driel, J. Wassermann, C. Pelties, A. Schiemenz, and H. Igel. Tilt effects on moment tensor inversion in the nearfield of active volcanoes. Geophys. J. Int., 202(3):1711-1721, 2015.

H. Vasyura-Bathke, J. Dettmer, R. Dutta, P. M. Mai, and S. Jónsson. Accounting for theory errors with empirical Bayesian noise models in nonlinear centroid moment tensor estimation. Geophys. J. Int., 225:1412-1431, 2021.

V. Vavrycǔk and S. G. Kim. Nonisotropic radiation of the 2013 North Korean nuclear explosion. Geophys. Res. Lett., 41:7048-7056, 2014.

A. Velikoseltsev, K. U. Schreiber, A. Yankovsky, J.-P. R. Wells, A. Boronachin, and A. Tkachenko. On the application of fiber optic gyroscopes for detection of seismic rotations. Journal of Seismology, 16:623-637, 2012.

J. Wassermann, S. Lehndorfer, H. Igel, and U. Schreiber. Performance test of a commercial rotational sensor. Bull. Seismol. Soc. Am., 99:1449-1456, 2009.

Y. Yagi and Y. Fukahata. Importance of covariance component in inversion analyses of densely sampled observed data: an application to waveform data inversion of seismic source processes. Geophys. J. Int, 175(1):215-221, 2008.

L. Zhou, W. Zhang, Y. Shen, X. Chen, and J. Zhang. Location and moment tensor inversion of small earthquakes using 3D Greens functions in models with rugged topography: application to the Longmenshan fault zone. Earthquake Science, 29(3):139-151, 2016. 